\title{
FURTHER IMPROVEMENTS OF CFA 3.0 BY COMBINING INPAINTING AND PANSHARPENING TECHNIQUES
}

\author{
Chiman Kwan and Jude Larkin \\ Applied Research, LLC, Rockville, Maryland, USA
}

\begin{abstract}
Color Filter Array (CFA) has been widely used in digital cameras. There are many variants of CFAs in the literature. Recently, a new CFA known as CFA 3.0 was proposed by us and has been shown to yield reasonable performance as compared to some standard ones. In this paper, we investigate the use of inpainting algorithms to further improve the demosaicing performance of CFA 3.0. Six conventional and deep learning based inpainting algorithms were compared. Extensive experiments demonstrated that one algorithm improved over other approaches.
\end{abstract}

\section{KEYWORDS}

CFA 3.0, color filter array, demosaicing, inpainting, deep learning, pansharpening

\section{INTRODUCTION}

Bayer pattern [1] was invented in the early 1980's and is still a very popular color filter array (CFA) for digital cameras. The Bayer pattern as shown in Figure 1(a) is also known as CFA 1.0 in the literature. Even for planetary explorations, NASA has adopted the Bayer pattern in the Mastcam imagers onboard the Mars rover Curiosity [2]-[5].

Aiming to improve the Bayer pattern in low lighting conditions, Kodak researchers $[6,7]$ invented a red-green-blue-white (RGBW) CFA pattern, which is also known as CFA 2.0, which is shown in Figure 1(b). Half of the pixels in CFA 2.0 are white and the remaining pixels share the R, G, and $\mathrm{B}$ colors. Due to the presence of white pixels, the camera sensitivity is increased and hence the performance of CFA 2.0 in low lighting conditions should be better than CFA 1.0. Extensive experiments in [8] showed that CFA 2.0 is in indeed better than CFA 1.0 in low lighting conditions, where Poisson noise is dominant. Some additional studies were also carried out for CFA 2.0 [9].

In a recent paper by us [12], a new CFA pattern known as CFA 3.0 was proposed. In CFA 3.0 as shown in Figure 1(c), even more white pixels are introduced, hoping that the demosaicing performance will be further improved in low lighting conditions. Unfortunately, having more white pixels means that fewer color pixels will be present in the color filter array. Consequently, the overall performance of CFA 3.0 for low lighting images is slightly inferior to CFA 2.0 but still better than CFA 1.0 [10][11]. 
Signal \& Image Processing: An International Journal (SIPIJ) Vol.11, No.6, December 2020

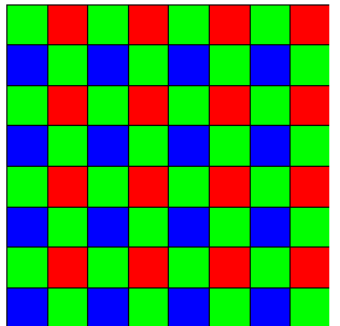

(a)

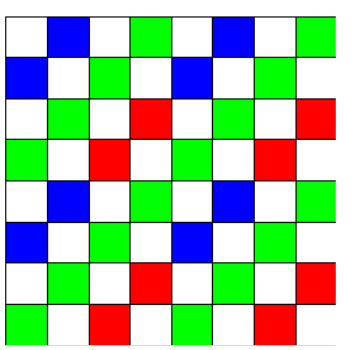

(b)

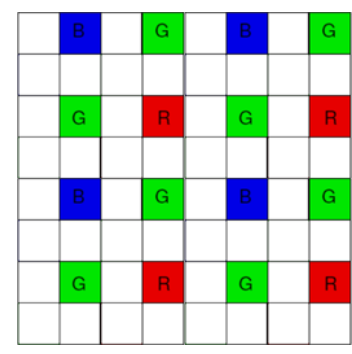

(c)

Figure 1. Three CFA patterns. (a) CFA 1.0; (b) CFA 2.0; (c) CFA 3.0.

In [12], we used an interpolation method known as local directional interpolation and nonlocal adaptive thresholding (LDI-NAT) [13] to create the luminance or panchromatic (pan) band. After that, the full resolution luminance band is then fused with the low resolution color image via pansharpening techniques to create the full resolution color image. The whole process is summarized in Figure 2. The luminance image is also termed the panchromatic image and we use them interchangeably in this paper.

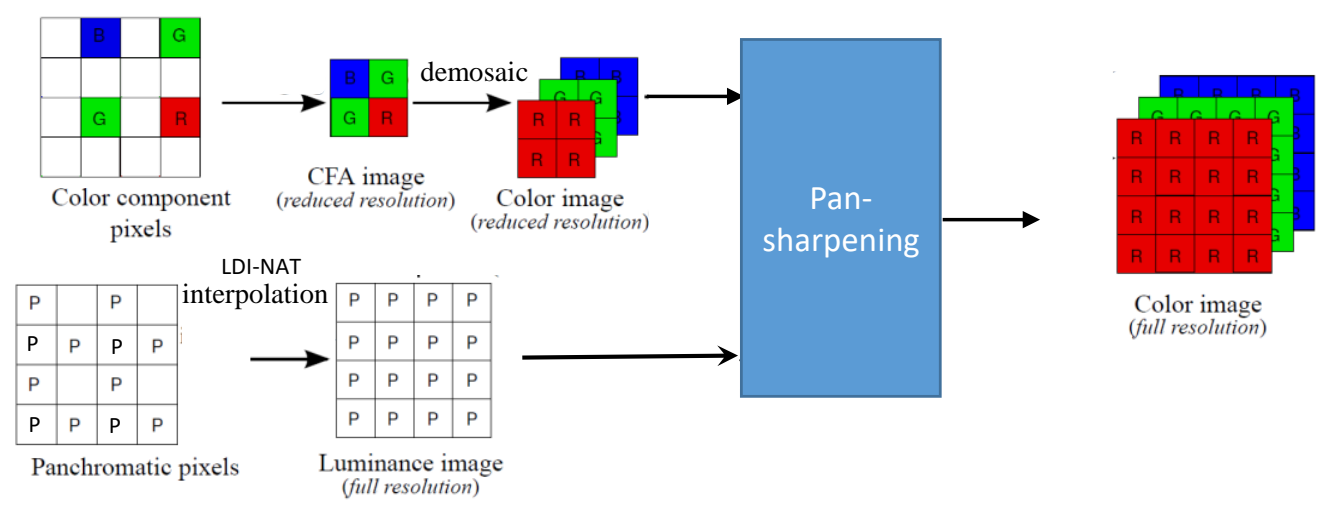

Figure 2. A pansharpening approach for CFA 3.0.

From Figure 2, it is natural to ask several research questions. First, are there any methods that can further enhance the performance of the luminance image? As we will see in the experiments, the demosaicing performance can be improved quite a lot if the ground truth panchromatic image is used. This means that if one can apply a high performing interpolation method to fill in the missing pixels in the panchromatic band, then the overall demosaicing performance will be increased. Second, if there does exist a good interpolation/inpainting algorithm, how much performance gain can we achieve?

In this paper, we will focus on answering the two aforementioned questions. In particular, we propose to investigate various inpainting methods to create the panchromatic band. In addition to the LDI-NAT method, we also applied five other methods, including conventional and deep learning algorithms. After the inpainting is done, we then apply various pansharpening algorithms to generate the final demosaiced images. We extensively evaluate the different combinations using the Kodak benchmark images.

There are three major contributions in this paper: 
Signal \& Image Processing: An International Journal (SIPIJ) Vol.11, No.6, December 2020

- We are the first ones to apply various inpainting methods to generate the pan band for demosaicing CFA 3.0.

- We are also the first team to investigate the combination of inpainting and pansharpening in demosaicing CFA 3.0.

- The combination of inpainting and pansharpening results are better than before, but there is still room for further improvement.

The rest of our paper is organized as follows. Section 2 summarizes the methods, data, and performance metrics. Section 3 presents all the experimental results. Finally, some concluding remarks and future directions will be given.

\section{Methods, Data, and Performance Metrics}

\subsection{Architecture of Demosaicing CFA 3.0 with Inpainting and Pansharpening}

In our earlier paper [12], we presented a standard approach to demosaicing CFA 3.0. For completeness, that architecture is depicted in Figure 3. The R, G, and B pixels in the CFA 3.0 are extracted to form a reduced resolution CFA image. A demosaicing algorithm (LDI-NAT) is used to demosaic it and generate a reduced resolution color image. Parallel to this activity, the white/panchromatic pixels in the CFA 3.0 are also interpolated to form the luminance image using the same LDI-NAT algorithm. The luminance image is then downsampled by two times via averaging and the reduced resolution luminance image is subtracted from the reduced resolution color image to generate the chrominance-luminance image. A simple upsampling via bicubic interpolation is then performed to generate the full resolution chrominance-luminance image. Finally, the luminance image and the chrominance-luminance image are added together to form the final demosaicing image. This simple architecture is very simple to understand and implement. Although there are many algorithms in the literature that could be used in the interpolation and demosaicing steps, we chose LDI-NAT in [12] simply because it has reasonable performance.

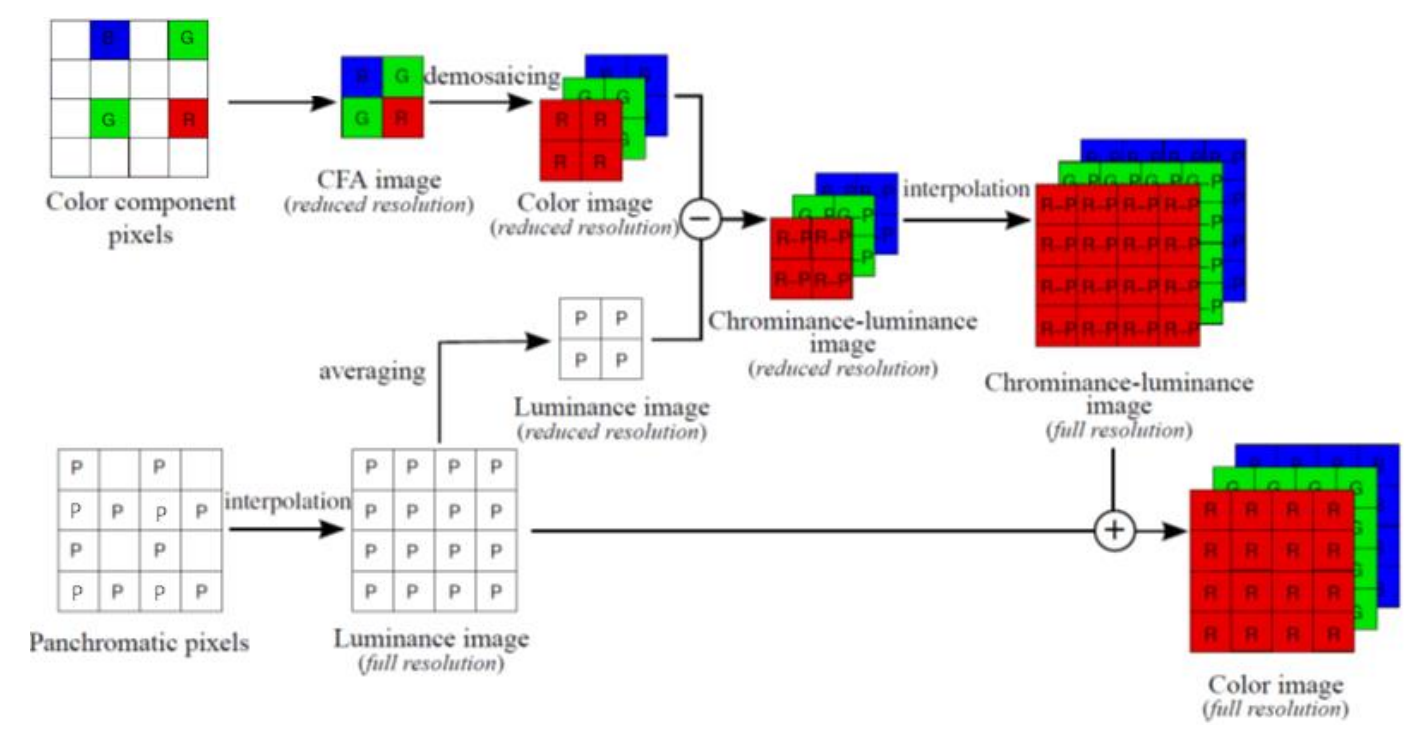

Figure 3. Standard demosaicing framework for CFA 3.0.

In this paper, we propose the architecture shown in Figure 4, which is essentially the same as the architecture shown in Figure 2 except the interpolation step. In Figure 4, we emphasize on the use 
Signal \& Image Processing: An International Journal (SIPIJ) Vol.11, No.6, December 2020

of inpainting algorithms for generating the luminance image and the other parts are exactly the same as Figure 2. The difference between interpolation and inpainting is very subtle. Normally, interpolation is used to fill in missing pixels in images that have regular missing patterns. On the other hand, inpainting is referring to missing pixels with free form patterns. That is, the missing patterns can have arbitrary shapes. As can be seen from Figure 4, the pansharpening step is to utilize the high resolution luminance image to sharpen the reduced resolution color image and the final pansharpened image will be the demosaiced image.

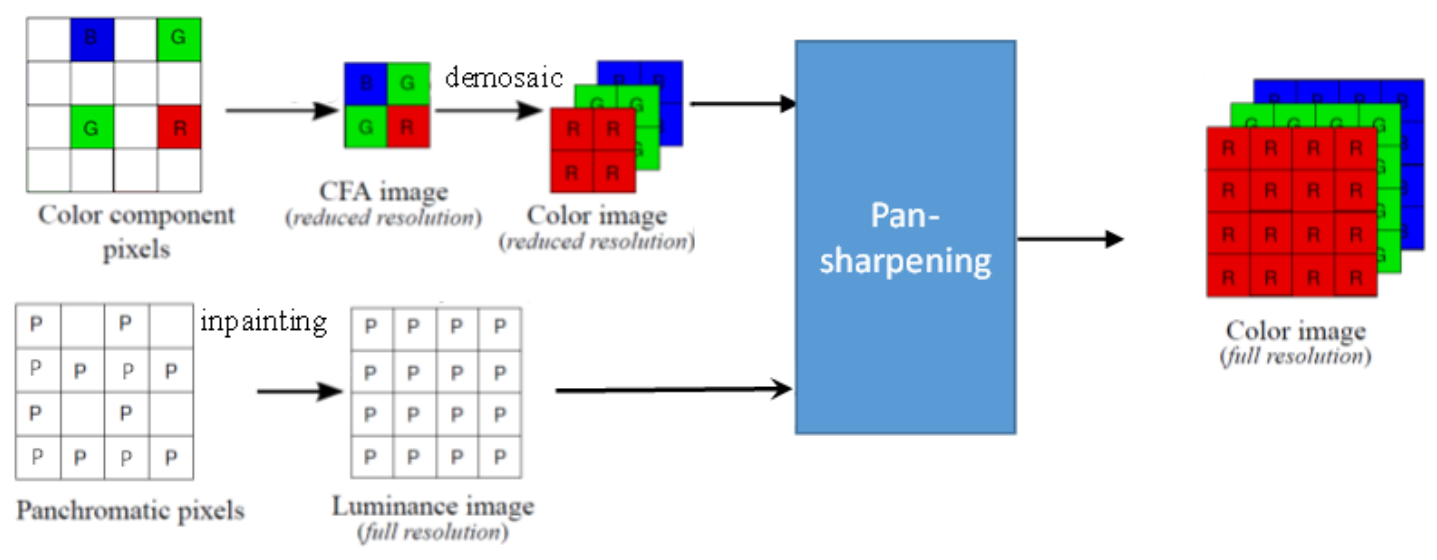

Figure 4. Proposed architecture for the combined inpainting and pansharpening demosaicing approach.

\subsection{Inpainting Methods}

In recent years, there are many new developments in inpainting. In this paper, we have evaluated the following six techniques:

- Linear Directional Interpolation and Nonlocal Adaptive Thresholding (LDI-NAT): This algorithm is a demosaicing algorithm. However, it can be used for both demosaicing as well as interpolation [13]. It has good performance in our earlier studies [8]. We used LDINAT in our earlier paper [12] and this will be the baseline for our inpainting investigations.

- Laplacian: This method [14] fills in each missing pixel using the Laplacian interpolation formula by finding the mean of the surrounding known values.

- Bilinear: This method simply uses the average of neighboring pixels to fill in the missing pixels. Bilinear and Laplacian have similar performance.

- Inpaint nans: We denote this as "inpaint" in our later experiments. This method was developed by D'Errico[15]. This is a very simple method that only uses the neighboring pixels to estimate the missing pixels which will be referred as NaNs (not a number).

- FOE: The Field of Experts method (FOE) was developed by Roth [16]. This method uses pre-trained models that are used to filter out noise and obstructions in images.

- Generative Inpainting (GenIn)[17]: A new inpainting method, Generative Inpainting (GenIn), which is a deep learning-based method [17], was considered in our research. It was developed at the University of Illinois that aims to outperform typical deep learning methods that use convolutional neural network (CNN) models. GenIn builds on CNN and Generative Adversarial Networks (GAN) in an effort to encourage cohesion between created and existing pixels. GenIn ranked the first in one Github page (https://github.com/1900zyh/Awesome-Image-Inpainting), which contains many conventional and deep learning based algorithms. This is the reason we chose GenIn in this paper. 
Signal \& Image Processing: An International Journal (SIPIJ) Vol.11, No.6, December 2020

\subsection{Pansharpening Methods}

In the paper [18] written by us, we proposed a pansharpening approach to demosaicing CFA 2.0. This approach is illustrated in Figure 4. The missing pixels in the panchromatic band are interpolated. At the same time, the reduced resolution CFA is demosaiced. We then apply pansharpening to generate the full resolution color image. There are many pansharpening algorithms that can be used. Principal Component Analysis (PCA) [19], Smoothing Filter-based Intensity Modulation (SFIM) [20], Modulation Transfer Function Generalized Laplacian Pyramid (GLP) [21], MTF-GLP with High Pass Modulation (HPM) [22], Gram Schmidt (GS) [23], GS Adaptive (GSA) [24], Guided Filter PCA (GFPCA) [25], PRACS [26] and hybrid color mapping (HCM) [27]-[31] have been used in our experiments. The list is a representative, if not exhaustive, set of competitive pansharpening algorithms.

\subsection{Data}

We downloaded a benchmark data set (Kodak) from a website (http://r0k.us/graphics/kodak/) and selected 12 images, which are shown in Figure 5. It should be noted that this dataset is wellknown and has been used by many authors in the demosaicing community such as [32]-[36]. These clean images will be used as reference images for objective performance metrics generation. Moreover, they will be used for generating noisy images that emulate low lighting conditions.

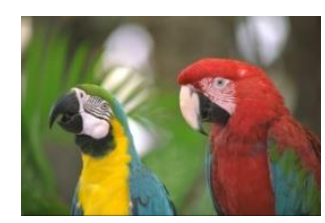

Image 1

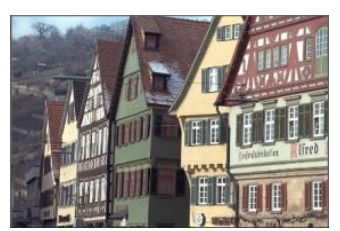

Image 4

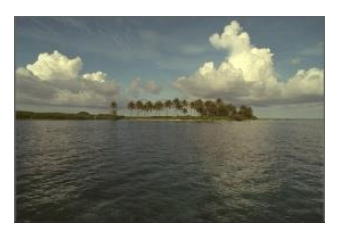

Image 7

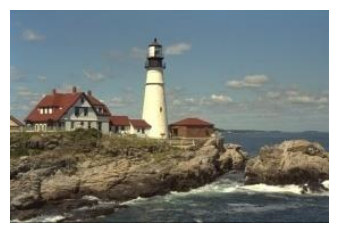

Image 10

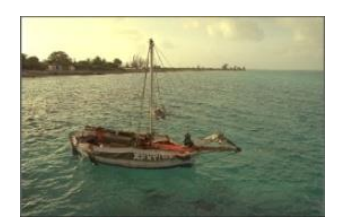

Image 2

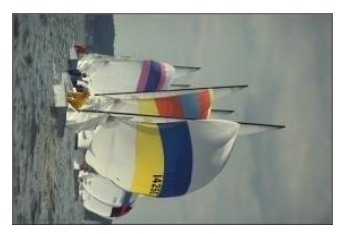

Image 5

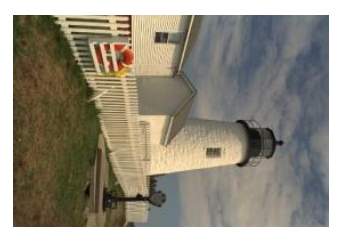

Image 8

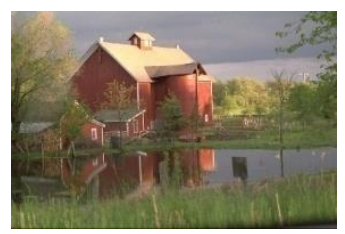

Image 11

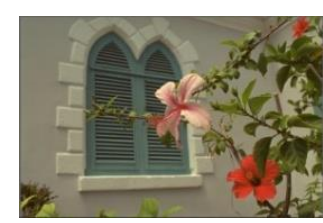

Image 3

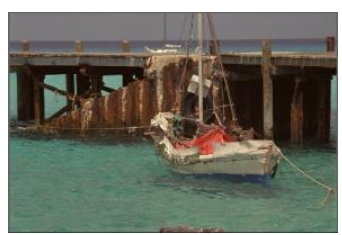

Image 6

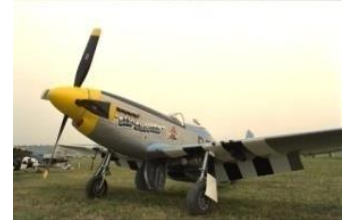

Image 9

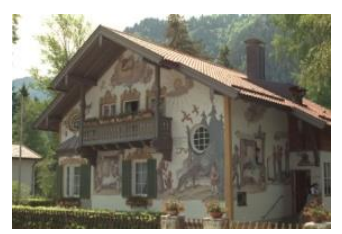

Image 12

Figure 5. Twelve clean images from the Kodak dataset. 
Signal \& Image Processing: An International Journal (SIPIJ) Vol.11, No.6, December 2020

\subsection{Metrics}

Five performance metrics were used in our experiments to compare the different methods and CFAs. These metrics are well-known in the literature.

- $\quad$ Peak Signal-to-Noise Ratio (PSNR) [37]

Separate PSNRs in dBs are computed for each band. A combined PSNR is the average of the PSNRs of the individual bands. Higher PSNR values imply higher image quality.

- Structural SIMilarity (SSIM)

In [38], SSIM was defined to measure the closeness between two images. An SSIM value of 1 means that the two images are the same.

- Human Visual System (HVS) metric

Details of HVS metric in $\mathrm{dB}$ can be found in [39]. Higher values imply better results.

- HVSm (HVS with masking) [40]

Similar to HVS, HVS incorporates the visual masking effects in computing the metrics. Higher values imply better results.

- CIELAB

We also used CIELAB [41] for assessing demosaicing and denoising performance in our experiments. Smaller values mean good results.

It should be noted that the HVS and HVSm have better correlation with human perceptions than the other three metrics [42][43].

\section{EXPERIMENTAL RESUltS}

In this section, we will first compare the performance of different inpainting algorithms on the generation of panchromatic bands. This step is critical for the overall performance of the demosaicing process. We will then focus on several case studies based on the performance of the inpainting results. In particular, we will generate the demosaicing results using the best inpainting method, the previous interpolation method of LDI-NAT in our earlier paper [12], and the ideal case of using the ground truth panchromatic band for inpainting.

\subsection{Comparing Different Inpaintingmethods for Pan Band Generation}

Here, we will focus on comparing the six different inpainting methods on each image from the KODAK dataset using the CFA3 pattern. For ease of exposition, we only used PSNR. The PSNR is calculated by comparing each inpainted result with the Ground Truth (Reference) pan image, which is generated by taking the average of the RGB bands in the original Kodak image. Table 1 summarizes the PSNR metrics of six inpainting algorithms using the 12 Kodak images. The missing pattern is the CFA 3.0 where $25 \%$ of the pixels in the panchromatic bands are missing. Figure 6 shows the averaged PSNR metrics of the five inpainting algorithms. We have the following observations:

- The method of LDI (LDI-NAT), which was used in our earlier paper [12], did not yield the best performance. It is $0.92 \mathrm{~dB}$ lower than the best performance algorithm (FOE).

- The deep learning method (GenIn) has a mediocre performance, which may be a little surprising because we had high expectation for it. We think that GenIn may be more 
Signal \& Image Processing: An International Journal (SIPIJ) Vol.11, No.6, December 2020

suitable for free-form missing clusters where big chunks of missing blocks with irregular shapes are present in images.

- FOE yielded the best performance, which is somewhat surprising because it was developed long time ago.

Table 1. Bilinear is the same as Laplacian. Inpainting results for 12 panchromatic images using five algorithms.

\begin{tabular}{|c|c|c|c|c|c|c|}
\hline & Inpaint & FOE & Laplace & LDI & Bilinear & Generative \\
\hline Img1 & 47.93 & 48.00 & 46.64 & 45.54 & 46.64 & 45.19 \\
\hline Img2 & 41.06 & 42.09 & 39.75 & 40.61 & 39.75 & 41.34 \\
\hline Img3 & 46.01 & 46.50 & 44.45 & 44.50 & 44.45 & 43.85 \\
\hline Img4 & 39.16 & 40.47 & 37.83 & 39.56 & 37.83 & 39.27 \\
\hline Img5 & 45.44 & 46.23 & 43.74 & 44.20 & 43.74 & 45.92 \\
\hline Img6 & 42.53 & 43.08 & 41.35 & 41.87 & 41.35 & 41.19 \\
\hline Img7 & 43.72 & 44.76 & 42.15 & 42.62 & 42.15 & 42.01 \\
\hline Img8 & 41.78 & 42.76 & 40.56 & 41.79 & 40.57 & 41.43 \\
\hline Img9 & 44.10 & 44.45 & 43.29 & 43.32 & 43.29 & 42.44 \\
\hline Img10 & 41.62 & 41.68 & 40.88 & 41.13 & 40.88 & 41.21 \\
\hline Img11 & 42.85 & 42.83 & 42.05 & 42.08 & 42.05 & 42.04 \\
\hline Img12 & 41.10 & 40.88 & 40.32 & 40.70 & 40.33 & 40.16 \\
\hline Average & 43.11 & 43.25 & 41.92 & 42.33 & 41.92 & 42.17 \\
\hline
\end{tabular}

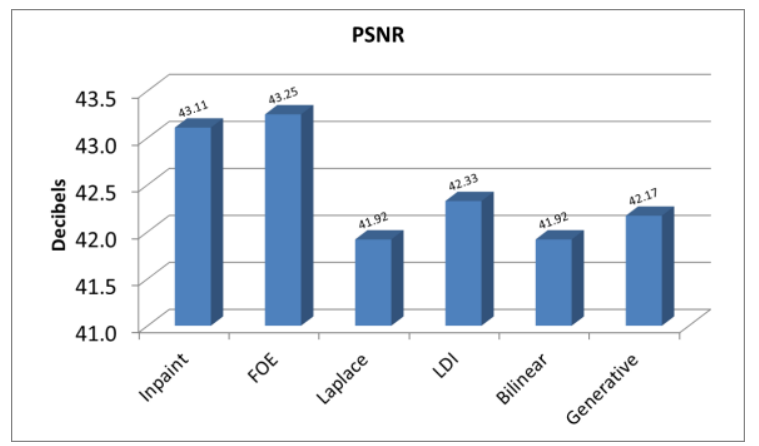

Figure 6. Averaged PSNR of five inpainting algorithms.

\subsection{Pansharpening Results using Different Inpaintingmethods}

In this section, we will summarize three representative case studies. First, we will summarize the demosaicing results of an earlier approach in which applied LDI-NAT for interpolation the pan band. This will form the baseline for comparisons. Second, we will summarize the demosaicing results using the best inpainting method (FOE) based on results in Section 3.1. Third, we will summarize the ideal demosaicing results where the ground truth pan images are used in the demosaicing process.

\subsubsection{LDI-NAT + Pansharpening}

Here, the pan images were generated using LDI-NAT. After that, 11 pansharpening algorithms were applied to demosaic the 12 Kodak images. Table 2 summarizes all the performance metrics 
Signal \& Image Processing: An International Journal (SIPIJ) Vol.11, No.6, December 2020

of those 12 images. For easier interpretation of those numbers in Table 2, Figure 7 shows the bar charts of the averaged performance metrics. In terms of PSNR, the best performing method is the GSA method. However, GFPCA achieved the highest performance in CIELAB, HVS, and HVSm. In particular, GFPCA is $3 \mathrm{dBs}$ better than all the other methods in terms HVS and HVSmand this is remarkable. The "standard" method has the best score in terms of SSIM. However, the difference is very small between the "standard" method and others.

As mentioned earlier in Section 2, the PSNR and SSIM metrics do not necessarily match well with human perception. This turns out to be indeed the case. From those images in Figure 8, one can easily conclude that the GFPCA results have less artifacts and look closer to the ground truth images. The HVS and HVSm metrics corroborate the above subjective evaluations results.

Table 2.Demosaicing results of Kodak images. LDI-NAT was used to generate the pan images.

\begin{tabular}{|c|c|c|c|c|c|c|c|c|c|c|c|c|c|}
\hline Image & & $\begin{array}{c}\text { Baselin } \\
\text { e }\end{array}$ & $\begin{array}{c}\text { Standar } \\
\text { d }\end{array}$ & GSA & НCM & $\begin{array}{c}\text { SFI } \\
\text { M }\end{array}$ & PCA & $\begin{array}{c}\text { GFPC } \\
\text { A }\end{array}$ & GLP & HРM & GS & $\begin{array}{c}\text { PRAC } \\
\text { S }\end{array}$ & $\begin{array}{c}\text { Best } \\
\text { Score }\end{array}$ \\
\hline \multirow[t]{5}{*}{ Img1 } & $\begin{array}{c}\text { PSN } \\
\mathrm{R}\end{array}$ & 31.936 & 33.894 & $\begin{array}{c}34.12 \\
6 \\
\end{array}$ & $\begin{array}{c}33.52 \\
6 \\
\end{array}$ & $\begin{array}{c}33.12 \\
3 \\
\end{array}$ & $\begin{array}{c}33.82 \\
8 \\
\end{array}$ & 33.158 & $\begin{array}{c}33.81 \\
0 \\
\end{array}$ & $\begin{array}{c}33.06 \\
6 \\
\end{array}$ & $\begin{array}{c}34.13 \\
0\end{array}$ & 33.466 & 34.130 \\
\hline & $\begin{array}{c}\text { Ciela } \\
\mathrm{b}\end{array}$ & 2.659 & 2.374 & 2.393 & 2.481 & 2.699 & 2.445 & 2.773 & 2.429 & 2.724 & 2.351 & 2.453 & 2.351 \\
\hline & SSIM & 0.739 & 0.859 & 0.854 & 0.831 & 0.838 & 0.845 & 0.806 & 0.853 & 0.834 & 0.858 & 0.820 & 0.859 \\
\hline & HVS & 28.233 & 27.610 & $\begin{array}{c}28.43 \\
8\end{array}$ & $\begin{array}{c}28.30 \\
9\end{array}$ & $\begin{array}{c}28.45 \\
3\end{array}$ & $\begin{array}{c}28.48 \\
1\end{array}$ & 27.947 & $\begin{array}{c}28.35 \\
3\end{array}$ & $\begin{array}{c}28.46 \\
8\end{array}$ & $\begin{array}{c}28.40 \\
3\end{array}$ & 28.407 & 28.481 \\
\hline & $\begin{array}{c}\text { HVS } \\
\mathrm{m}\end{array}$ & 29.767 & 29.031 & $\begin{array}{c}29.88 \\
1 \\
\end{array}$ & $\begin{array}{c}29.83 \\
0 \\
\end{array}$ & $\begin{array}{c}29.97 \\
5 \\
\end{array}$ & $\begin{array}{c}29.93 \\
4\end{array}$ & 28.834 & $\begin{array}{c}29.82 \\
2\end{array}$ & $\begin{array}{c}29.98 \\
3\end{array}$ & $\begin{array}{c}29.83 \\
6 \\
\end{array}$ & 29.868 & 29.983 \\
\hline \multirow[t]{5}{*}{ Img2 } & $\begin{array}{c}\text { PSN } \\
\text { R }\end{array}$ & 26.771 & 30.585 & $\begin{array}{c}30.58 \\
1 \\
\end{array}$ & $\begin{array}{c}30.21 \\
1 \\
\end{array}$ & $\begin{array}{c}29.91 \\
8 \\
\end{array}$ & $\begin{array}{c}30.40 \\
6 \\
\end{array}$ & 30.136 & $\begin{array}{c}30.08 \\
9 \\
\end{array}$ & $\begin{array}{c}29.89 \\
6 \\
\end{array}$ & $\begin{array}{c}30.52 \\
1\end{array}$ & 30.230 & 30.585 \\
\hline & $\begin{array}{c}\text { Ciela } \\
\text { b }\end{array}$ & 4.860 & 3.766 & 3.803 & 3.874 & 3.901 & 3.891 & 3.219 & 3.906 & 3.904 & 3.830 & 3.903 & 3.219 \\
\hline & SSIM & 0.685 & 0.868 & 0.867 & 0.856 & 0.856 & 0.845 & 0.823 & 0.857 & 0.853 & 0.850 & 0.852 & 0.868 \\
\hline & HVS & 23.976 & 24.331 & $\begin{array}{c}24.48 \\
6\end{array}$ & $\begin{array}{c}24.30 \\
4\end{array}$ & $\begin{array}{c}24.26 \\
4\end{array}$ & $\begin{array}{c}24.72 \\
6\end{array}$ & 27.637 & $\begin{array}{c}24.39 \\
8\end{array}$ & $\begin{array}{c}24.22 \\
8\end{array}$ & $\begin{array}{c}24.40 \\
3\end{array}$ & 24.445 & 27.637 \\
\hline & $\begin{array}{c}\mathrm{HVS} \\
\mathrm{m}\end{array}$ & 25.515 & 25.629 & $\begin{array}{c}25.80 \\
3 \\
\end{array}$ & $\begin{array}{c}25.66 \\
5\end{array}$ & $\begin{array}{c}25.66 \\
6\end{array}$ & $\begin{array}{c}26.07 \\
6\end{array}$ & 29.840 & $\begin{array}{c}25.72 \\
1\end{array}$ & $\begin{array}{c}25.61 \\
3\end{array}$ & $\begin{array}{c}25.70 \\
3\end{array}$ & 25.762 & 29.840 \\
\hline \multirow[t]{5}{*}{ Img3 } & $\begin{array}{c}\text { PSN } \\
\mathrm{R} \\
\end{array}$ & 30.815 & 33.017 & $\begin{array}{c}32.99 \\
7 \\
\end{array}$ & $\begin{array}{c}32.15 \\
6 \\
\end{array}$ & $\begin{array}{c}32.42 \\
0 \\
\end{array}$ & $\begin{array}{c}32.99 \\
5 \\
\end{array}$ & 34.055 & $\begin{array}{c}32.69 \\
8 \\
\end{array}$ & $\begin{array}{c}32.39 \\
5 \\
\end{array}$ & $\begin{array}{c}33.03 \\
7 \\
\end{array}$ & 32.648 & 34.055 \\
\hline & $\begin{array}{c}\text { Ciela } \\
b\end{array}$ & 3.758 & 3.378 & 3.313 & 3.535 & 3.432 & 3.324 & 2.949 & 3.345 & 3.459 & 3.303 & 3.398 & 2.949 \\
\hline & SSIM & 0.786 & $\begin{array}{l}0.888 \\
\end{array}$ & 0.884 & 0.870 & 0.879 & 0.877 & 0.873 & 0.878 & 0.873 & 0.877 & 0.870 & 0.888 \\
\hline & HVS & 27.087 & 27.099 & $\begin{array}{c}27.26 \\
6 \\
\end{array}$ & $\begin{array}{c}27.08 \\
1\end{array}$ & $\begin{array}{c}27.21 \\
1\end{array}$ & $\begin{array}{c}27.40 \\
3\end{array}$ & 29.897 & $\begin{array}{c}27.19 \\
2 \\
\end{array}$ & $\begin{array}{c}27.21 \\
8\end{array}$ & $\begin{array}{c}27.36 \\
6\end{array}$ & 27.221 & 29.897 \\
\hline & $\begin{array}{c}\text { HVS } \\
\mathrm{m}\end{array}$ & 28.861 & 28.734 & $\begin{array}{c}28.92 \\
8 \\
\end{array}$ & $\begin{array}{c}28.89 \\
4 \\
\end{array}$ & $\begin{array}{c}28.97 \\
6 \\
\end{array}$ & $\begin{array}{c}29.06 \\
5 \\
\end{array}$ & 31.435 & $\begin{array}{c}28.87 \\
0 \\
\end{array}$ & $\begin{array}{c}28.97 \\
3 \\
\end{array}$ & $\begin{array}{c}29.02 \\
3 \\
\end{array}$ & 28.900 & 31.435 \\
\hline \multirow[t]{5}{*}{ Img4 } & $\begin{array}{c}\text { PSN } \\
\text { R }\end{array}$ & 22.762 & 26.980 & $\begin{array}{c}27.49 \\
6 \\
\end{array}$ & $\begin{array}{c}27.09 \\
0 \\
\end{array}$ & $\begin{array}{c}26.80 \\
8 \\
\end{array}$ & $\begin{array}{c}26.88 \\
4 \\
\end{array}$ & 26.873 & $\begin{array}{c}26.76 \\
2 \\
\end{array}$ & $\begin{array}{c}26.77 \\
1 \\
\end{array}$ & $\begin{array}{c}26.88 \\
4 \\
\end{array}$ & 26.933 & 27.496 \\
\hline & $\begin{array}{c}\text { Ciela } \\
\mathrm{b}\end{array}$ & 7.484 & 5.434 & 5.327 & 5.178 & 5.314 & 5.662 & 4.841 & 5.664 & 5.364 & 5.644 & 5.371 & 4.841 \\
\hline & SSIM & 0.752 & 0.925 & 0.925 & 0.919 & 0.915 & 0.901 & 0.891 & 0.913 & 0.911 & 0.903 & 0.913 & 0.925 \\
\hline & HVS & 20.315 & 20.370 & $\begin{array}{c}21.07 \\
7\end{array}$ & $\begin{array}{c}21.06 \\
4\end{array}$ & $\begin{array}{c}21.16 \\
0\end{array}$ & $\begin{array}{c}20.98 \\
6\end{array}$ & 24.117 & $\begin{array}{c}21.09 \\
5\end{array}$ & $\begin{array}{c}21.20 \\
3\end{array}$ & $\begin{array}{c}20.86 \\
7\end{array}$ & 20.918 & 24.117 \\
\hline & $\begin{array}{c}\mathrm{HVS} \\
\mathrm{m}\end{array}$ & 21.997 & 21.682 & $\begin{array}{c}22.47 \\
6\end{array}$ & $\begin{array}{c}22.52 \\
6\end{array}$ & $\begin{array}{c}22.65 \\
6\end{array}$ & $\begin{array}{c}22.37 \\
7\end{array}$ & 26.303 & $\begin{array}{c}22.54 \\
7 \\
\end{array}$ & $\begin{array}{c}22.70 \\
6\end{array}$ & $\begin{array}{c}22.24 \\
0\end{array}$ & 22.337 & 26.303 \\
\hline \multirow[t]{5}{*}{ Img5 } & $\begin{array}{c}\text { PSN } \\
\text { R }\end{array}$ & 30.816 & 34.107 & $\begin{array}{c}33.95 \\
2 \\
\end{array}$ & $\begin{array}{c}33.68 \\
6 \\
\end{array}$ & $\begin{array}{c}33.55 \\
8 \\
\end{array}$ & $\begin{array}{c}33.82 \\
5\end{array}$ & 34.541 & $\begin{array}{c}33.69 \\
4 \\
\end{array}$ & $\begin{array}{c}33.46 \\
9\end{array}$ & $\begin{array}{c}34.13 \\
2\end{array}$ & 33.766 & 34.541 \\
\hline & $\begin{array}{c}\text { Ciela } \\
\text { b }\end{array}$ & 2.568 & 2.100 & 2.172 & 2.054 & 2.107 & 2.180 & 1.914 & 2.132 & 2.123 & 2.070 & 2.136 & 1.914 \\
\hline & SSIM & 0.668 & 0.868 & 0.852 & 0.859 & 0.859 & 0.845 & 0.798 & 0.855 & 0.852 & 0.859 & 0.838 & 0.868 \\
\hline & HVS & 27.733 & 27.824 & $\begin{array}{c}28.15 \\
5 \\
\end{array}$ & $\begin{array}{c}28.08 \\
3 \\
\end{array}$ & $\begin{array}{c}28.08 \\
3 \\
\end{array}$ & $\begin{array}{c}28.34 \\
4 \\
\end{array}$ & 30.514 & $\begin{array}{c}28.15 \\
4 \\
\end{array}$ & $\begin{array}{c}28.08 \\
3 \\
\end{array}$ & $\begin{array}{c}28.13 \\
2 \\
\end{array}$ & 28.146 & 30.514 \\
\hline & $\begin{array}{c}\text { HVS } \\
\mathrm{m}\end{array}$ & 29.444 & 29.335 & $\begin{array}{c}29.70 \\
7 \\
\end{array}$ & $\begin{array}{c}29.67 \\
6\end{array}$ & $\begin{array}{c}29.77 \\
2 \\
\end{array}$ & $\begin{array}{c}29.91 \\
2\end{array}$ & 32.085 & $\begin{array}{c}29.75 \\
0\end{array}$ & $\begin{array}{c}29.77 \\
5\end{array}$ & $\begin{array}{c}29.66 \\
2\end{array}$ & 29.688 & 32.085 \\
\hline \multirow[t]{5}{*}{ Img6 } & $\begin{array}{c}\text { PSN } \\
\mathrm{R} \\
\end{array}$ & 27.706 & 30.874 & $\begin{array}{c}31.03 \\
1 \\
\end{array}$ & $\begin{array}{c}30.39 \\
1\end{array}$ & $\begin{array}{c}30.38 \\
2 \\
\end{array}$ & $\begin{array}{c}30.98 \\
0\end{array}$ & 31.381 & $\begin{array}{c}30.64 \\
7 \\
\end{array}$ & $\begin{array}{c}30.27 \\
8 \\
\end{array}$ & $\begin{array}{c}30.92 \\
6 \\
\end{array}$ & 30.601 & 31.381 \\
\hline & $\begin{array}{c}\text { Ciela } \\
\text { b }\end{array}$ & 5.555 & 4.605 & 4.721 & 4.528 & 4.464 & 4.657 & 3.797 & 4.619 & 4.544 & 4.698 & 4.575 & 3.797 \\
\hline & SSIM & 0.711 & 0.896 & 0.879 & 0.877 & 0.881 & 0.864 & 0.848 & 0.882 & 0.873 & 0.860 & 0.869 & 0.896 \\
\hline & HVS & 24.678 & 24.823 & $\begin{array}{c}25.11 \\
4\end{array}$ & $\begin{array}{c}24.87 \\
7\end{array}$ & $\begin{array}{c}25.03 \\
1 \\
\end{array}$ & $\begin{array}{c}25.03 \\
4\end{array}$ & 27.599 & $\begin{array}{c}25.15 \\
9 \\
\end{array}$ & $\begin{array}{c}25.04 \\
7\end{array}$ & $\begin{array}{c}25.09 \\
7\end{array}$ & 24.967 & 27.599 \\
\hline & $\begin{array}{c}\text { HVS } \\
\mathrm{m}\end{array}$ & 26.353 & 26.293 & $\begin{array}{c}26.60 \\
6\end{array}$ & $\begin{array}{c}26.47 \\
0\end{array}$ & $\begin{array}{c}26.60 \\
3\end{array}$ & $\begin{array}{c}26.52 \\
0\end{array}$ & 29.306 & $\begin{array}{c}26.66 \\
5\end{array}$ & $\begin{array}{c}26.61 \\
1\end{array}$ & $\begin{array}{c}26.59 \\
1\end{array}$ & 26.495 & 29.306 \\
\hline \multirow[t]{2}{*}{ Img7 } & $\begin{array}{c}\text { PSN } \\
\text { R }\end{array}$ & 30.446 & 34.517 & $\begin{array}{c}34.46 \\
9\end{array}$ & $\begin{array}{c}34.08 \\
1\end{array}$ & $\begin{array}{c}33.70 \\
1\end{array}$ & $\begin{array}{c}34.35 \\
1\end{array}$ & 33.767 & $\begin{array}{c}33.91 \\
7\end{array}$ & $\begin{array}{c}33.68 \\
0\end{array}$ & $\begin{array}{c}34.39 \\
1\end{array}$ & 34.183 & 34.517 \\
\hline & Ciela & 3.639 & 2.751 & 2.773 & 2.809 & 2.855 & 2.799 & 2.501 & 2.854 & 2.857 & 2.785 & 2.841 & 2.501 \\
\hline
\end{tabular}


Signal \& Image Processing: An International Journal (SIPIJ) Vol.11, No.6, December 2020

\begin{tabular}{|c|c|c|c|c|c|c|c|c|c|c|c|c|c|}
\hline & $\mathrm{b}$ & & & & & & & & & & & & \\
\hline & SSIM & 0.731 & 0.904 & 0.903 & 0.896 & 0.894 & 0.897 & 0.853 & 0.894 & 0.891 & 0.897 & 0.892 & 0.904 \\
\hline & HVS & 27.968 & 28.395 & $\begin{array}{c}28.40 \\
9\end{array}$ & $\begin{array}{c}28.32 \\
3\end{array}$ & $\begin{array}{c}28.19 \\
9\end{array}$ & $\begin{array}{c}28.49 \\
7\end{array}$ & 32.017 & $\begin{array}{c}28.32 \\
1\end{array}$ & $\begin{array}{c}28.15 \\
9\end{array}$ & $\begin{array}{c}28.41 \\
1\end{array}$ & 28.415 & 32.017 \\
\hline & $\begin{array}{c}\mathrm{HVS} \\
\mathrm{m}\end{array}$ & 29.538 & 29.687 & $\begin{array}{c}29.69 \\
6\end{array}$ & $\begin{array}{c}29.66 \\
1\end{array}$ & $\begin{array}{c}29.57 \\
9\end{array}$ & $\begin{array}{c}29.80 \\
0\end{array}$ & 34.461 & $\begin{array}{c}29.62 \\
8\end{array}$ & $\begin{array}{c}29.51 \\
7\end{array}$ & $\begin{array}{c}29.70 \\
6\end{array}$ & 29.727 & 34.461 \\
\hline \multirow[t]{5}{*}{ Img8 } & $\begin{array}{c}\text { PSN } \\
\mathrm{R} \\
\end{array}$ & 26.939 & 30.748 & $\begin{array}{c}31.07 \\
8\end{array}$ & $\begin{array}{c}30.41 \\
9\end{array}$ & $\begin{array}{c}30.25 \\
3\end{array}$ & $\begin{array}{c}30.56 \\
8\end{array}$ & 30.319 & $\begin{array}{c}30.39 \\
0\end{array}$ & $\begin{array}{c}30.12 \\
3 \\
\end{array}$ & $\begin{array}{c}30.67 \\
0\end{array}$ & 30.439 & 31.078 \\
\hline & $\begin{array}{c}\text { Ciela } \\
\text { b }\end{array}$ & 4.697 & 3.707 & 3.566 & 3.769 & 3.704 & 3.758 & 3.200 & 3.746 & 3.734 & 3.707 & 3.812 & 3.200 \\
\hline & SSIM & 0.733 & 0.900 & 0.899 & 0.885 & 0.890 & 0.883 & 0.860 & 0.891 & 0.886 & 0.888 & 0.877 & 0.900 \\
\hline & HVS & 24.460 & 24.087 & $\begin{array}{c}25.01 \\
3\end{array}$ & $\begin{array}{c}24.85 \\
4\end{array}$ & $\begin{array}{c}24.96 \\
9\end{array}$ & $\begin{array}{c}25.03 \\
9\end{array}$ & 28.845 & $\begin{array}{c}25.00 \\
4\end{array}$ & $\begin{array}{c}24.99 \\
6\end{array}$ & $\begin{array}{c}24.86 \\
6\end{array}$ & 24.883 & 28.845 \\
\hline & $\begin{array}{c}\mathrm{HVS} \\
\mathrm{m}\end{array}$ & 26.141 & 25.461 & $\begin{array}{c}26.45 \\
3\end{array}$ & $\begin{array}{c}26.37 \\
8\end{array}$ & $\begin{array}{c}26.49 \\
6\end{array}$ & $\begin{array}{c}26.47 \\
3\end{array}$ & 30.948 & $\begin{array}{c}26.47 \\
6\end{array}$ & $\begin{array}{c}26.51 \\
9\end{array}$ & $\begin{array}{c}26.27 \\
7\end{array}$ & 26.335 & 30.948 \\
\hline \multirow[t]{5}{*}{ Img9 } & $\begin{array}{c}\text { PSN } \\
\mathrm{R} \\
\end{array}$ & 29.775 & 32.268 & $\begin{array}{c}32.68 \\
2 \\
\end{array}$ & $\begin{array}{c}32.11 \\
7\end{array}$ & $\begin{array}{c}31.74 \\
2 \\
\end{array}$ & $\begin{array}{c}32.67 \\
6 \\
\end{array}$ & 33.783 & $\begin{array}{c}32.31 \\
6 \\
\end{array}$ & $\begin{array}{c}31.66 \\
9 \\
\end{array}$ & $\begin{array}{c}32.66 \\
8 \\
\end{array}$ & 32.318 & 33.783 \\
\hline & $\begin{array}{c}\text { Ciela } \\
\text { b }\end{array}$ & 3.062 & 2.705 & 2.592 & 2.601 & 2.911 & 2.561 & 2.202 & 2.669 & 2.974 & 2.566 & 2.595 & 2.202 \\
\hline & SSIM & 0.508 & 0.634 & 0.637 & 0.623 & 0.623 & 0.582 & 0.615 & 0.577 & 0.564 & 0.582 & 0.616 & 0.637 \\
\hline & HVS & 26.329 & 26.028 & $\begin{array}{c}26.75 \\
3\end{array}$ & $\begin{array}{c}26.63 \\
2\end{array}$ & $\begin{array}{c}26.80 \\
8\end{array}$ & $\begin{array}{c}26.74 \\
8\end{array}$ & 30.150 & $\begin{array}{c}26.82 \\
3\end{array}$ & $\begin{array}{c}26.82 \\
0\end{array}$ & $\begin{array}{c}26.77 \\
9\end{array}$ & 26.621 & 30.150 \\
\hline & $\begin{array}{c}\mathrm{HVS} \\
\mathrm{m}\end{array}$ & 27.955 & 27.482 & $\begin{array}{c}28.23 \\
4\end{array}$ & $\begin{array}{c}28.18 \\
1\end{array}$ & $\begin{array}{c}28.36 \\
2\end{array}$ & $\begin{array}{c}28.22 \\
8\end{array}$ & 31.987 & $\begin{array}{c}28.33 \\
1\end{array}$ & $\begin{array}{c}28.36 \\
5\end{array}$ & $\begin{array}{c}28.26 \\
4\end{array}$ & 28.115 & 31.987 \\
\hline \multirow[t]{5}{*}{$\operatorname{Img} 10$} & $\begin{array}{c}\text { PSN } \\
\text { R } \\
\end{array}$ & 27.054 & 30.354 & $\begin{array}{c}30.54 \\
7 \\
\end{array}$ & $\begin{array}{c}29.97 \\
0 \\
\end{array}$ & $\begin{array}{c}29.88 \\
5 \\
\end{array}$ & $\begin{array}{c}30.35 \\
0 \\
\end{array}$ & 31.177 & $\begin{array}{c}30.01 \\
4 \\
\end{array}$ & $\begin{array}{c}29.82 \\
2 \\
\end{array}$ & $\begin{array}{c}30.30 \\
9 \\
\end{array}$ & 30.118 & 31.177 \\
\hline & $\begin{array}{c}\text { Ciela } \\
\text { b }\end{array}$ & 4.808 & 3.975 & 3.930 & 3.927 & 3.915 & 3.991 & 3.223 & 4.075 & 3.940 & 3.959 & 3.936 & 3.223 \\
\hline & SSIM & 0.687 & 0.867 & 0.867 & 0.856 & 0.857 & 0.832 & 0.802 & 0.858 & 0.853 & 0.855 & 0.848 & 0.867 \\
\hline & HVS & 24.184 & 24.135 & $\begin{array}{c}24.51 \\
7\end{array}$ & $\begin{array}{c}24.44 \\
0\end{array}$ & $\begin{array}{c}24.45 \\
9\end{array}$ & $\begin{array}{c}24.45 \\
0\end{array}$ & 28.393 & $\begin{array}{c}24.50 \\
8\end{array}$ & $\begin{array}{c}24.44 \\
1\end{array}$ & $\begin{array}{c}24.51 \\
5\end{array}$ & 24.458 & 28.393 \\
\hline & $\begin{array}{c}\text { HVS } \\
\mathrm{m}\end{array}$ & 25.796 & 25.521 & $\begin{array}{c}25.92 \\
8\end{array}$ & $\begin{array}{c}25.96 \\
3\end{array}$ & $\begin{array}{c}25.96 \\
9\end{array}$ & $\begin{array}{c}25.86 \\
7\end{array}$ & 30.357 & $\begin{array}{c}25.93 \\
1\end{array}$ & $\begin{array}{c}25.93 \\
6 \\
\end{array}$ & $\begin{array}{c}25.93 \\
5\end{array}$ & 25.910 & 30.357 \\
\hline \multirow[t]{5}{*}{$\operatorname{Img} 11$} & $\begin{array}{c}\text { PSN } \\
\text { R }\end{array}$ & 29.027 & 32.011 & $\begin{array}{c}32.23 \\
4\end{array}$ & $\begin{array}{c}31.70 \\
3\end{array}$ & $\begin{array}{c}31.70 \\
7\end{array}$ & $\begin{array}{c}32.12 \\
1\end{array}$ & 31.682 & $\begin{array}{c}31.83 \\
5\end{array}$ & $\begin{array}{c}31.65 \\
5\end{array}$ & $\begin{array}{c}32.14 \\
3\end{array}$ & 31.687 & 32.234 \\
\hline & $\begin{array}{c}\text { Ciela } \\
\text { b }\end{array}$ & 4.282 & 3.556 & 3.529 & 3.654 & 3.606 & 3.545 & 3.412 & 3.628 & 3.627 & 3.543 & 3.605 & 3.412 \\
\hline & SSIM & 0.722 & 0.882 & 0.883 & 0.866 & 0.875 & 0.875 & 0.840 & 0.875 & 0.871 & 0.876 & 0.862 & 0.883 \\
\hline & HVS & 26.763 & 26.320 & $\begin{array}{c}27.14 \\
3 \\
\end{array}$ & $\begin{array}{c}27.13 \\
4 \\
\end{array}$ & $\begin{array}{c}27.17 \\
5 \\
\end{array}$ & $\begin{array}{c}27.08 \\
0\end{array}$ & 28.744 & $\begin{array}{c}27.17 \\
7 \\
\end{array}$ & $\begin{array}{c}27.21 \\
5 \\
\end{array}$ & $\begin{array}{c}27.08 \\
5 \\
\end{array}$ & 27.089 & 28.744 \\
\hline & $\begin{array}{c}\text { HVS } \\
\mathrm{m}\end{array}$ & 28.417 & 27.778 & $\begin{array}{c}28.62 \\
6\end{array}$ & $\begin{array}{c}28.70 \\
8\end{array}$ & $\begin{array}{c}28.72 \\
4\end{array}$ & $\begin{array}{c}28.54 \\
8\end{array}$ & 30.402 & $\begin{array}{c}28.69 \\
3\end{array}$ & $\begin{array}{c}28.76 \\
2\end{array}$ & $\begin{array}{c}28.55 \\
2\end{array}$ & 28.586 & 30.402 \\
\hline \multirow[t]{5}{*}{ Img12 } & $\begin{array}{c}\text { PSN } \\
\text { R }\end{array}$ & 25.845 & 28.451 & $\begin{array}{c}29.11 \\
5 \\
\end{array}$ & $\begin{array}{c}28.77 \\
9 \\
\end{array}$ & $\begin{array}{c}28.76 \\
9 \\
\end{array}$ & $\begin{array}{c}28.79 \\
6 \\
\end{array}$ & 29.171 & $\begin{array}{c}28.80 \\
7 \\
\end{array}$ & $\begin{array}{c}28.73 \\
3 \\
\end{array}$ & $\begin{array}{c}28.78 \\
2 \\
\end{array}$ & 28.712 & 29.171 \\
\hline & $\begin{array}{c}\text { Ciela } \\
\text { b }\end{array}$ & 4.525 & 3.669 & 3.558 & 3.610 & 3.621 & 3.786 & 3.176 & 3.707 & 3.649 & 3.783 & 3.620 & 3.176 \\
\hline & SSIM & 0.770 & 0.909 & 0.910 & 0.903 & 0.902 & 0.880 & 0.883 & 0.891 & 0.889 & 0.880 & 0.902 & 0.910 \\
\hline & HVS & 24.168 & 23.290 & $\begin{array}{c}24.59 \\
0\end{array}$ & $\begin{array}{c}24.67 \\
4\end{array}$ & $\begin{array}{c}24.65 \\
8\end{array}$ & $\begin{array}{c}24.38 \\
4\end{array}$ & 27.561 & $\begin{array}{c}24.58 \\
4\end{array}$ & $\begin{array}{c}24.65 \\
2\end{array}$ & $\begin{array}{c}24.38 \\
5\end{array}$ & 24.521 & 27.561 \\
\hline & $\begin{array}{c}\mathrm{HVS} \\
\mathrm{m}\end{array}$ & 25.807 & 24.728 & $\begin{array}{c}26.10 \\
0\end{array}$ & $\begin{array}{c}26.21 \\
9\end{array}$ & $\begin{array}{c}26.22 \\
7\end{array}$ & $\begin{array}{c}25.84 \\
2\end{array}$ & 29.625 & $\begin{array}{c}26.11 \\
5\end{array}$ & $\begin{array}{c}26.21 \\
1\end{array}$ & $\begin{array}{c}25.84 \\
3\end{array}$ & 26.026 & 29.625 \\
\hline \multirow[t]{5}{*}{$\begin{array}{c}\text { e } \\
\end{array}$} & $\begin{array}{c}\text { PSN } \\
\text { R }\end{array}$ & 28.324 & 31.484 & $\begin{array}{c}31.69 \\
2\end{array}$ & $\begin{array}{c}31.17 \\
8\end{array}$ & $\begin{array}{c}31.02 \\
2\end{array}$ & $\begin{array}{c}31.48 \\
2\end{array}$ & 31.670 & $\begin{array}{c}31.24 \\
8\end{array}$ & $\begin{array}{c}30.96 \\
3\end{array}$ & $\begin{array}{c}31.55 \\
0\end{array}$ & 31.258 & 31.692 \\
\hline & $\begin{array}{c}\text { Ciela } \\
\text { b }\end{array}$ & 4.325 & 3.502 & 3.473 & 3.502 & 3.544 & 3.550 & 3.101 & 3.564 & 3.575 & 3.520 & 3.520 & 3.101 \\
\hline & SSIM & 0.708 & 0.867 & 0.863 & 0.853 & 0.856 & 0.844 & 0.824 & 0.852 & 0.846 & 0.849 & 0.846 & 0.867 \\
\hline & HVS & 25.491 & 25.359 & $\begin{array}{c}25.91 \\
3\end{array}$ & $\begin{array}{c}25.81 \\
5\end{array}$ & $\begin{array}{c}25.87 \\
2\end{array}$ & $\begin{array}{c}25.93 \\
1\end{array}$ & 28.618 & $\begin{array}{c}25.89 \\
7\end{array}$ & $\begin{array}{c}25.87 \\
8\end{array}$ & $\begin{array}{c}25.85 \\
9\end{array}$ & 25.841 & 28.618 \\
\hline & $\begin{array}{c}\text { HVS } \\
\mathrm{m}\end{array}$ & 27.132 & 26.780 & $\begin{array}{c}27.37 \\
0\end{array}$ & $\begin{array}{c}27.34 \\
7\end{array}$ & $\begin{array}{c}27.41 \\
7\end{array}$ & $\begin{array}{c}27.38 \\
7\end{array}$ & 30.465 & $\begin{array}{c}27.37 \\
9\end{array}$ & $\begin{array}{c}27.41 \\
4 \\
\end{array}$ & $\begin{array}{c}27.30 \\
3\end{array}$ & 27.312 & 30.465 \\
\hline
\end{tabular}


Signal \& Image Processing: An International Journal (SIPIJ) Vol.11, No.6, December 2020

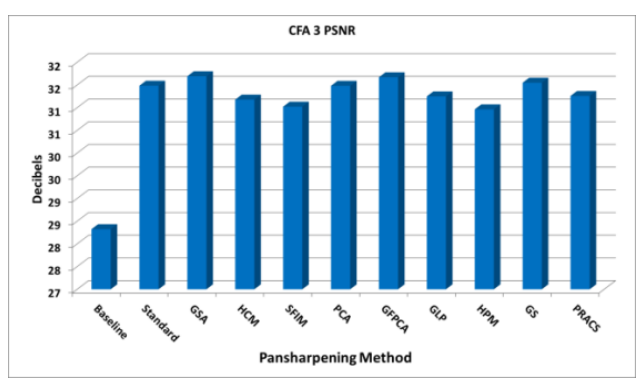

(a) PSNR

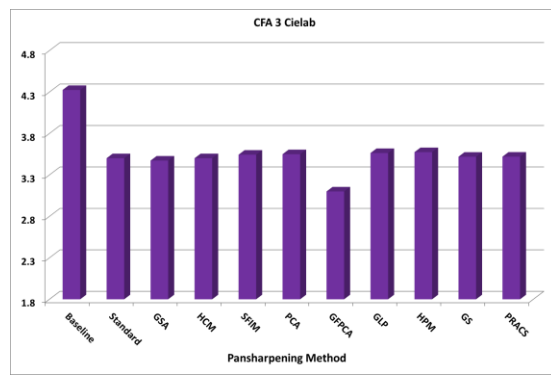

(b) CIELAB

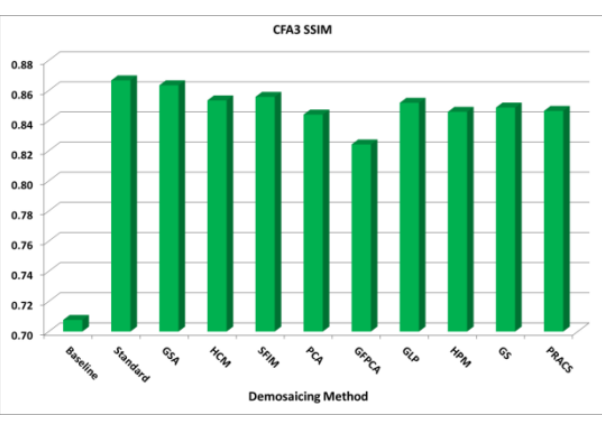

(c) SSIM

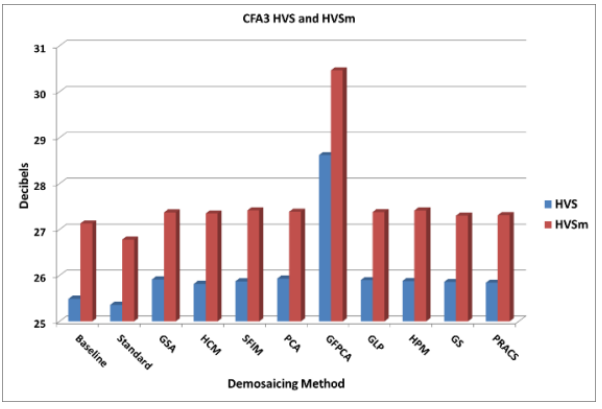

(d) HVS and HVSm

Figure 7. Averaged performance metrics of all the demosaicing results for the LDI-DAT case.

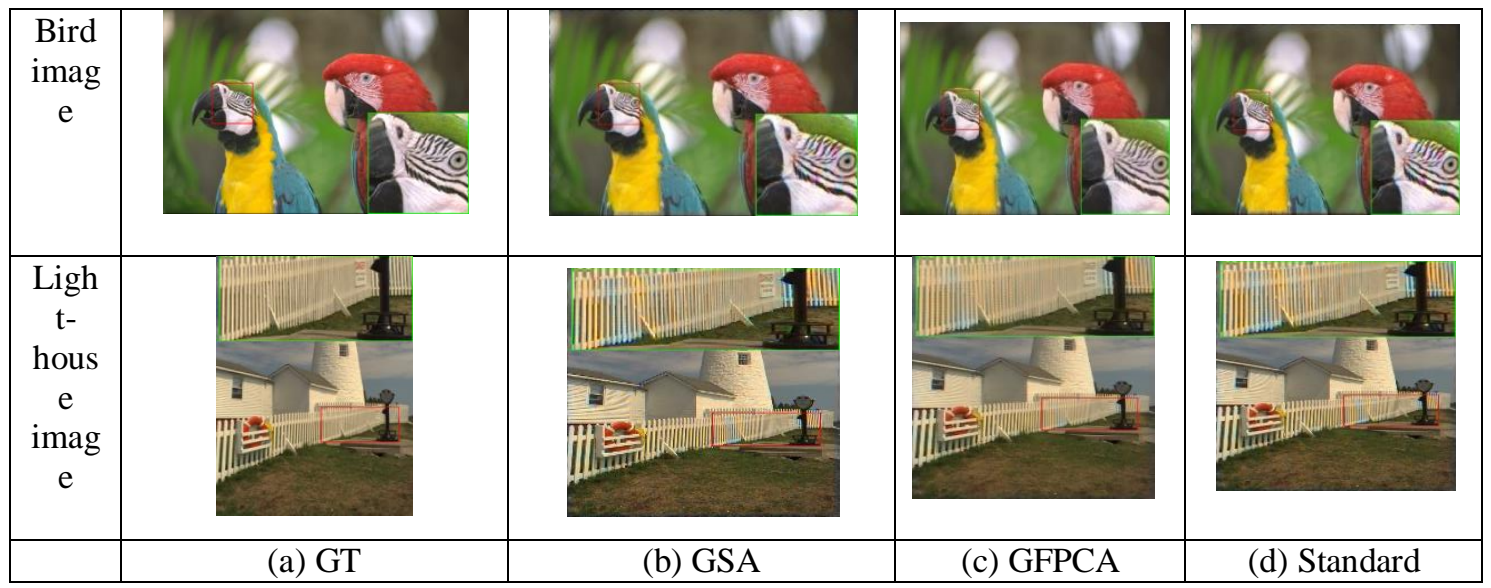

Figure 8. The ground truth image and three selected demosaiced images using the LDI-NAT inpainting method for pan band.

\subsubsection{FOE + Pansharpening}

In Section 3.1, we observed that the FOE algorithm yielded the best inpainting performance. Here, we show the demosaicing results of the 12 Kodak images by a combination of FOE and various pansharpening algorithms. Table 3 summarizes all the performance metrics of those 12 images. Figure 7 shows the bar charts of the averaged performance metrics. It can be seen that GFPCA achieved the highest performance in PSNR, CIELAB, HVS, and HVSm. Similar to the LDI-NAT case, GFPCA is $3 \mathrm{dBs}$ better than all the other methods in terms HVS and HVSm. From those images in Figure 10, one can easily conclude that the GFPCA results have less artifacts and look closer to the ground truth images. 
Signal \& Image Processing: An International Journal (SIPIJ) Vol.11, No.6, December 2020

Table 3.Demosaicing results of Kodak images. FOE was used to generate the pan images.

\begin{tabular}{|c|c|c|c|c|c|c|c|c|c|c|c|c|c|}
\hline Image & & $\begin{array}{c}\text { Baselin } \\
\text { e }\end{array}$ & $\begin{array}{c}\text { Standar } \\
\text { d }\end{array}$ & GSA & HCM & SFIM & PCA & $\begin{array}{c}\text { GFPC } \\
\text { A }\end{array}$ & GLP & HPM & GS & $\begin{array}{c}\text { PRAC } \\
\mathrm{S}\end{array}$ & $\begin{array}{r}\text { Best } \\
\text { Score } \\
\end{array}$ \\
\hline \multirow[t]{5}{*}{ Img 1} & PSNR & 32.004 & 33.988 & $\begin{array}{c}34.24 \\
1\end{array}$ & $\begin{array}{c}33.62 \\
1\end{array}$ & $\begin{array}{c}33.15 \\
0\end{array}$ & $\begin{array}{c}33.99 \\
0\end{array}$ & 33.320 & $\begin{array}{c}33.89 \\
7\end{array}$ & $\begin{array}{c}33.09 \\
0\end{array}$ & $\begin{array}{c}34.29 \\
5\end{array}$ & 33.591 & $\begin{array}{c}34.29 \\
5\end{array}$ \\
\hline & $\begin{array}{c}\text { Ciela } \\
\text { b }\end{array}$ & 2.651 & 2.347 & 2.387 & 2.460 & 2.700 & 2.412 & 2.756 & 2.408 & 2.728 & 2.321 & 2.434 & 2.321 \\
\hline & SSIM & 0.741 & 0.858 & 0.851 & 0.830 & 0.833 & 0.847 & 0.807 & 0.849 & 0.829 & $\begin{array}{l}0.858 \\
\end{array}$ & 0.821 & 0.858 \\
\hline & HVS & 28.147 & 27.283 & $\begin{array}{c}28.23 \\
9\end{array}$ & $\begin{array}{c}28.06 \\
4\end{array}$ & $\begin{array}{c}28.20 \\
6\end{array}$ & $\begin{array}{c}28.29 \\
1\end{array}$ & 28.034 & $\begin{array}{c}28.09 \\
6\end{array}$ & $\begin{array}{c}28.18 \\
3\end{array}$ & $\begin{array}{c}28.22 \\
0\end{array}$ & 28.264 & $\begin{array}{c}28.29 \\
1\end{array}$ \\
\hline & $\begin{array}{c}\text { HVS } \\
\mathrm{m}\end{array}$ & 29.690 & 28.717 & $\begin{array}{c}29.69 \\
4\end{array}$ & $\begin{array}{c}29.60 \\
6\end{array}$ & $\begin{array}{c}29.74 \\
1\end{array}$ & $\begin{array}{c}29.75 \\
6\end{array}$ & 28.878 & $\begin{array}{c}29.58 \\
9\end{array}$ & $\begin{array}{c}29.72 \\
0\end{array}$ & $\begin{array}{c}29.66 \\
5\end{array}$ & 29.735 & $\begin{array}{c}29.75 \\
6\end{array}$ \\
\hline \multirow[t]{5}{*}{ Img2 } & PSNR & 26.836 & 30.908 & $\begin{array}{c}30.85 \\
5 \\
\end{array}$ & $\begin{array}{c}30.44 \\
6 \\
\end{array}$ & $\begin{array}{c}30.11 \\
2 \\
\end{array}$ & $\begin{array}{c}30.74 \\
7 \\
\end{array}$ & 30.393 & $\begin{array}{c}30.11 \\
9\end{array}$ & $\begin{array}{c}29.87 \\
6 \\
\end{array}$ & $\begin{array}{c}30.86 \\
7 \\
\end{array}$ & 30.569 & $\begin{array}{c}30.90 \\
8\end{array}$ \\
\hline & $\begin{array}{c}\text { Ciela } \\
\text { b }\end{array}$ & 4.834 & 3.636 & 3.693 & 3.745 & 3.791 & 3.751 & 3.145 & 3.848 & 3.845 & 3.688 & 3.777 & 3.145 \\
\hline & SSIM & 0.690 & 0.879 & 0.879 & 0.867 & 0.865 & 0.858 & 0.831 & 0.863 & 0.860 & 0.862 & 0.865 & 0.879 \\
\hline & HVS & 23.926 & 24.215 & $\begin{array}{c}24.32 \\
2\end{array}$ & $\begin{array}{c}24.12 \\
5\end{array}$ & $\begin{array}{c}24.02 \\
3\end{array}$ & $\begin{array}{c}24.57 \\
7\end{array}$ & 27.851 & $\begin{array}{c}24.11 \\
0\end{array}$ & $\begin{array}{c}23.87 \\
7\end{array}$ & $\begin{array}{c}24.25 \\
3\end{array}$ & 24.344 & $\begin{array}{c}27.85 \\
1\end{array}$ \\
\hline & $\begin{array}{c}\text { HVS } \\
\mathrm{m}\end{array}$ & 25.455 & 25.458 & $\begin{array}{c}25.61 \\
3 \\
\end{array}$ & $\begin{array}{c}25.45 \\
9 \\
\end{array}$ & $\begin{array}{c}25.39 \\
8 \\
\end{array}$ & $\begin{array}{c}25.88 \\
6 \\
\end{array}$ & 29.896 & $\begin{array}{c}25.43 \\
4 \\
\end{array}$ & $\begin{array}{c}25.27 \\
0 \\
\end{array}$ & $\begin{array}{c}25.51 \\
9 \\
\end{array}$ & 25.615 & $\begin{array}{c}29.89 \\
6 \\
\end{array}$ \\
\hline \multirow[t]{5}{*}{ Img3 } & PSNR & 30.836 & 33.074 & $\begin{array}{c}32.98 \\
2\end{array}$ & $\begin{array}{c}32.14 \\
7\end{array}$ & $\begin{array}{c}32.42 \\
4\end{array}$ & $\begin{array}{c}33.11 \\
2\end{array}$ & 34.350 & $\begin{array}{c}32.72 \\
3\end{array}$ & $\begin{array}{c}32.31 \\
2\end{array}$ & $\begin{array}{c}33.15 \\
3\end{array}$ & 32.656 & $\begin{array}{c}34.35 \\
0\end{array}$ \\
\hline & $\begin{array}{c}\text { Ciela } \\
\text { b }\end{array}$ & 3.748 & 3.328 & 3.316 & 3.492 & 3.434 & 3.243 & 2.901 & 3.292 & 3.469 & 3.223 & 3.376 & 2.901 \\
\hline & SSIM & 0.788 & 0.887 & 0.880 & 0.869 & 0.877 & 0.881 & 0.875 & 0.877 & 0.871 & 0.881 & 0.869 & 0.887 \\
\hline & HVS & 26.942 & 26.720 & $\begin{array}{c}26.87 \\
7\end{array}$ & $\begin{array}{c}26.63 \\
5\end{array}$ & $\begin{array}{c}26.78 \\
9\end{array}$ & $\begin{array}{c}27.04 \\
8\end{array}$ & 30.071 & $\begin{array}{c}26.79 \\
4\end{array}$ & $\begin{array}{c}26.75 \\
2\end{array}$ & $\begin{array}{c}27.01 \\
5\end{array}$ & 26.941 & $\begin{array}{c}30.07 \\
1\end{array}$ \\
\hline & $\begin{array}{c}\mathrm{HVS} \\
\mathrm{m}\end{array}$ & 28.725 & 28.377 & $\begin{array}{c}28.56 \\
4\end{array}$ & $\begin{array}{c}28.47 \\
9\end{array}$ & $\begin{array}{c}28.57 \\
7\end{array}$ & $\begin{array}{c}28.73 \\
1\end{array}$ & 31.491 & $\begin{array}{c}28.50 \\
5\end{array}$ & $\begin{array}{c}28.54 \\
1\end{array}$ & $\begin{array}{c}28.69 \\
4\end{array}$ & 28.639 & $\begin{array}{c}31.49 \\
1\end{array}$ \\
\hline \multirow[t]{5}{*}{ Img4 } & PSNR & 22.785 & 27.014 & $\begin{array}{c}27.53 \\
0\end{array}$ & $\begin{array}{c}27.12 \\
4\end{array}$ & $\begin{array}{c}26.80 \\
6\end{array}$ & $\begin{array}{c}26.93 \\
8\end{array}$ & 26.852 & $\begin{array}{c}26.69 \\
6\end{array}$ & $\begin{array}{c}26.67 \\
7\end{array}$ & $\begin{array}{c}26.93 \\
9\end{array}$ & 27.069 & $\begin{array}{c}27.53 \\
0\end{array}$ \\
\hline & $\begin{array}{c}\text { Ciela } \\
\text { b }\end{array}$ & 7.453 & 5.324 & 5.249 & 5.054 & 5.216 & 5.552 & 4.810 & 5.638 & 5.315 & 5.534 & 5.246 & 4.810 \\
\hline & SSIM & 0.754 & 0.930 & 0.929 & 0.923 & 0.919 & 0.907 & 0.894 & 0.916 & 0.914 & 0.908 & 0.918 & 0.930 \\
\hline & HVS & 20.284 & 20.307 & $\begin{array}{c}21.03 \\
8\end{array}$ & $\begin{array}{c}20.98 \\
2\end{array}$ & $\begin{array}{c}21.08 \\
0\end{array}$ & $\begin{array}{c}20.94 \\
1\end{array}$ & 24.156 & $\begin{array}{c}21.02 \\
7\end{array}$ & $\begin{array}{c}21.07 \\
8\end{array}$ & $\begin{array}{c}20.82 \\
3\end{array}$ & 20.890 & $\begin{array}{c}24.15 \\
6\end{array}$ \\
\hline & $\begin{array}{c}\mathrm{HVS} \\
\mathrm{m}\end{array}$ & 21.962 & 21.621 & $\begin{array}{c}22.42 \\
4 \\
\end{array}$ & $\begin{array}{c}22.44 \\
4 \\
\end{array}$ & $\begin{array}{c}22.56 \\
5 \\
\end{array}$ & $\begin{array}{c}22.31 \\
2 \\
\end{array}$ & 26.236 & $\begin{array}{c}22.47 \\
4 \\
\end{array}$ & $\begin{array}{c}22.57 \\
8 \\
\end{array}$ & $\begin{array}{c}22.17 \\
7 \\
\end{array}$ & 22.287 & $\begin{array}{c}26.23 \\
6 \\
\end{array}$ \\
\hline \multirow[t]{5}{*}{ Img5 } & PSNR & 30.856 & 34.218 & $\begin{array}{c}33.82 \\
1\end{array}$ & $\begin{array}{c}33.78 \\
5 \\
\end{array}$ & $\begin{array}{c}33.58 \\
8 \\
\end{array}$ & $\begin{array}{c}33.98 \\
0 \\
\end{array}$ & 34.732 & $\begin{array}{c}33.67 \\
5 \\
\end{array}$ & $\begin{array}{c}33.42 \\
0\end{array}$ & $\begin{array}{c}34.30 \\
5 \\
\end{array}$ & 33.835 & $\begin{array}{c}34.73 \\
2 \\
\end{array}$ \\
\hline & $\begin{array}{c}\text { Ciela } \\
\text { b }\end{array}$ & 2.559 & 2.075 & 2.240 & 2.027 & 2.105 & 2.136 & 1.892 & 2.134 & 2.134 & 2.025 & 2.142 & 1.892 \\
\hline & SSIM & 0.670 & 0.863 & 0.840 & 0.853 & 0.850 & 0.844 & 0.798 & 0.846 & 0.843 & 0.857 & 0.832 & 0.863 \\
\hline & HVS & 27.696 & 27.709 & $\begin{array}{c}28.05 \\
0\end{array}$ & $\begin{array}{c}28.01 \\
7\end{array}$ & $\begin{array}{c}27.96 \\
4\end{array}$ & $\begin{array}{c}28.30 \\
9\end{array}$ & 30.640 & $\begin{array}{c}28.06 \\
8\end{array}$ & $\begin{array}{c}27.92 \\
4\end{array}$ & $\begin{array}{c}28.09 \\
7\end{array}$ & 28.107 & $\begin{array}{c}30.64 \\
0\end{array}$ \\
\hline & $\begin{array}{c}\text { HVS } \\
\text { m }\end{array}$ & 29.403 & 29.199 & $\begin{array}{c}29.58 \\
5\end{array}$ & $\begin{array}{c}29.60 \\
1\end{array}$ & $\begin{array}{c}29.65 \\
2 \\
\end{array}$ & $\begin{array}{c}29.86 \\
4 \\
\end{array}$ & 32.091 & $\begin{array}{c}29.66 \\
0 \\
\end{array}$ & $\begin{array}{c}29.62 \\
7\end{array}$ & $\begin{array}{c}29.61 \\
3 \\
\end{array}$ & 29.629 & $\begin{array}{c}32.09 \\
1\end{array}$ \\
\hline \multirow[t]{5}{*}{ Img6 } & PSNR & 27.731 & 30.919 & $\begin{array}{c}31.04 \\
6\end{array}$ & $\begin{array}{c}30.40 \\
1\end{array}$ & $\begin{array}{c}30.34 \\
8\end{array}$ & $\begin{array}{c}31.09 \\
6\end{array}$ & 31.422 & $\begin{array}{c}30.59 \\
7\end{array}$ & $\begin{array}{c}30.13 \\
8\end{array}$ & $\begin{array}{c}31.03 \\
1\end{array}$ & 30.702 & $\begin{array}{c}31.42 \\
2\end{array}$ \\
\hline & $\begin{array}{c}\text { Ciela } \\
\text { b }\end{array}$ & 5.532 & 4.540 & 4.814 & 4.458 & 4.441 & 4.505 & 3.772 & 4.588 & 4.562 & 4.551 & 4.531 & 3.772 \\
\hline & SSIM & 0.716 & 0.903 & 0.880 & 0.884 & 0.885 & 0.878 & 0.853 & 0.885 & 0.874 & 0.874 & 0.875 & 0.903 \\
\hline & HVS & 24.581 & 24.559 & $\begin{array}{c}24.92 \\
9\end{array}$ & $\begin{array}{c}24.60 \\
8\end{array}$ & $\begin{array}{c}24.78 \\
6\end{array}$ & $\begin{array}{c}24.86 \\
8\end{array}$ & 27.678 & $\begin{array}{c}24.94 \\
2\end{array}$ & $\begin{array}{c}24.75 \\
5\end{array}$ & $\begin{array}{c}24.92 \\
2\end{array}$ & 24.830 & $\begin{array}{c}27.67 \\
8\end{array}$ \\
\hline & $\begin{array}{c}\text { HVS } \\
\text { m }\end{array}$ & 26.256 & 26.028 & $\begin{array}{c}26.41 \\
0\end{array}$ & $\begin{array}{c}26.22 \\
5\end{array}$ & $\begin{array}{c}26.36 \\
8\end{array}$ & $\begin{array}{c}26.34 \\
3\end{array}$ & 29.303 & $\begin{array}{c}26.44 \\
9\end{array}$ & $\begin{array}{c}26.33 \\
7\end{array}$ & $\begin{array}{c}26.40 \\
5\end{array}$ & 26.348 & $\begin{array}{c}29.30 \\
3\end{array}$ \\
\hline \multirow[t]{5}{*}{ Img7 } & PSNR & 30.510 & 34.925 & $\begin{array}{c}34.82 \\
0\end{array}$ & $\begin{array}{c}34.37 \\
6\end{array}$ & $\begin{array}{c}33.90 \\
2\end{array}$ & $\begin{array}{c}34.78 \\
8\end{array}$ & 34.014 & $\begin{array}{c}33.96 \\
2\end{array}$ & $\begin{array}{c}33.64 \\
8\end{array}$ & $\begin{array}{c}34.82 \\
7\end{array}$ & 34.604 & $\begin{array}{c}34.92 \\
5\end{array}$ \\
\hline & $\begin{array}{c}\text { Ciela } \\
\text { b }\end{array}$ & 3.618 & 2.651 & 2.683 & 2.705 & 2.770 & 2.686 & 2.454 & 2.815 & 2.814 & 2.669 & 2.738 & 2.454 \\
\hline & SSIM & 0.736 & 0.912 & 0.910 & 0.904 & 0.901 & 0.907 & $\begin{array}{l}2.759 \\
0.859\end{array}$ & 0.899 & 0.896 & 0.908 & 0.901 & 0.912 \\
\hline & HVS & 27.928 & 28.319 & $\begin{array}{c}28.31 \\
3\end{array}$ & $\begin{array}{c}28.14 \\
8\end{array}$ & $\begin{array}{c}27.99 \\
2\end{array}$ & $\begin{array}{c}28.42 \\
0\end{array}$ & 32.321 & $\begin{array}{c}28.11 \\
1\end{array}$ & $\begin{array}{c}27.82 \\
2\end{array}$ & $\begin{array}{c}28.33 \\
3\end{array}$ & 28.377 & $\begin{array}{c}32.32 \\
1\end{array}$ \\
\hline & $\begin{array}{c}\text { HVS } \\
\mathrm{m}\end{array}$ & 29.493 & 29.546 & $\begin{array}{c}29.54 \\
2\end{array}$ & $\begin{array}{c}29.45 \\
7\end{array}$ & $\begin{array}{c}29.33 \\
3\end{array}$ & $\begin{array}{c}29.65 \\
8\end{array}$ & 34.585 & $\begin{array}{c}29.38 \\
8\end{array}$ & $\begin{array}{c}29.17 \\
2\end{array}$ & $\begin{array}{c}29.56 \\
5\end{array}$ & 29.622 & $\begin{array}{c}34.58 \\
5\end{array}$ \\
\hline \multirow[t]{5}{*}{ Img8 } & PSNR & 26.974 & 30.834 & $\begin{array}{c}31.21 \\
9\end{array}$ & $\begin{array}{c}30.49 \\
3\end{array}$ & $\begin{array}{c}30.30 \\
9\end{array}$ & $\begin{array}{c}30.79 \\
7\end{array}$ & 30.476 & $\begin{array}{c}30.40 \\
2 \\
\end{array}$ & $\begin{array}{c}30.10 \\
4\end{array}$ & $\begin{array}{c}30.90 \\
0 \\
\end{array}$ & 30.598 & $\begin{array}{c}31.21 \\
9\end{array}$ \\
\hline & $\begin{array}{c}\text { Ciela } \\
\text { b }\end{array}$ & 4.685 & 3.669 & 3.517 & 3.725 & 3.669 & 3.681 & 3.179 & 3.728 & 3.714 & 3.626 & 3.761 & 3.179 \\
\hline & SSIM & 0.735 & 0.900 & 0.900 & 0.886 & 0.889 & 0.887 & 0.860 & 0.890 & 0.884 & 0.892 & 0.879 & 0.900 \\
\hline & HVS & 24.405 & 23.909 & $\begin{array}{c}24.90 \\
4\end{array}$ & $\begin{array}{c}24.70 \\
4\end{array}$ & $\begin{array}{c}24.81 \\
8\end{array}$ & $\begin{array}{c}24.95 \\
4\end{array}$ & 28.973 & $\begin{array}{c}24.85 \\
0\end{array}$ & $\begin{array}{c}24.80 \\
9\end{array}$ & $\begin{array}{c}24.78 \\
7\end{array}$ & 24.798 & $\begin{array}{c}28.97 \\
3\end{array}$ \\
\hline & $\begin{array}{c}\text { HVS } \\
\text { m }\end{array}$ & 26.086 & 25.295 & $\begin{array}{c}26.35 \\
7\end{array}$ & $\begin{array}{c}26.25 \\
9\end{array}$ & $\begin{array}{c}26.36 \\
5\end{array}$ & $\begin{array}{c}26.40 \\
0\end{array}$ & 30.940 & $\begin{array}{c}26.35 \\
3\end{array}$ & $\begin{array}{c}26.37 \\
0\end{array}$ & $\begin{array}{c}26.21 \\
1\end{array}$ & 26.246 & $\begin{array}{c}30.94 \\
0\end{array}$ \\
\hline Img9 & PSNR & 29.800 & 32.236 & 32.67 & 32.07 & 31.57 & 32.73 & 33.914 & 32.25 & 31.44 & 32.72 & 32.338 & 33.91 \\
\hline
\end{tabular}


Signal \& Image Processing: An International Journal (SIPIJ) Vol.11, No.6, December 2020

\begin{tabular}{|c|c|c|c|c|c|c|c|c|c|c|c|c|c|}
\hline & & & & 2 & 8 & 5 & 5 & & 5 & 7 & 8 & & 4 \\
\hline & $\begin{array}{c}\text { Ciela } \\
\text { b }\end{array}$ & 3.052 & 2.674 & 2.587 & 2.570 & 2.927 & 2.506 & 2.174 & 2.648 & 2.998 & 2.506 & 2.576 & 2.174 \\
\hline & SSIM & 0.511 & 0.634 & 0.637 & 0.623 & 0.622 & 0.587 & 0.615 & 0.578 & 0.563 & 0.588 & 0.621 & 0.637 \\
\hline & HVS & 26.255 & 25.799 & $\begin{array}{c}26.58 \\
6\end{array}$ & $\begin{array}{c}26.41 \\
0\end{array}$ & $\begin{array}{c}26.60 \\
4\end{array}$ & $\begin{array}{c}26.59 \\
7\end{array}$ & 30.276 & $\begin{array}{c}26.65 \\
2\end{array}$ & $\begin{array}{c}26.58 \\
5\end{array}$ & $\begin{array}{c}26.62 \\
9\end{array}$ & 26.500 & $\begin{array}{c}30.27 \\
6\end{array}$ \\
\hline & $\begin{array}{c}\mathrm{HVS} \\
\mathrm{m}\end{array}$ & 27.881 & 27.266 & $\begin{array}{c}28.06 \\
2\end{array}$ & $\begin{array}{c}27.97 \\
8\end{array}$ & $\begin{array}{c}28.16 \\
1\end{array}$ & $\begin{array}{c}28.07 \\
3\end{array}$ & 31.999 & $\begin{array}{c}28.16 \\
7\end{array}$ & $\begin{array}{c}28.14 \\
1\end{array}$ & $\begin{array}{c}28.11 \\
0\end{array}$ & 27.988 & $\begin{array}{c}31.99 \\
9\end{array}$ \\
\hline \multirow[t]{5}{*}{$\operatorname{Img} 10$} & PSNR & 27.087 & 30.381 & $\begin{array}{c}30.56 \\
4\end{array}$ & $\begin{array}{c}29.94 \\
4\end{array}$ & $\begin{array}{c}29.83 \\
9\end{array}$ & $\begin{array}{c}30.47 \\
4\end{array}$ & 31.298 & $\begin{array}{c}29.92 \\
8\end{array}$ & $\begin{array}{c}29.66 \\
3\end{array}$ & $\begin{array}{c}30.42 \\
7\end{array}$ & 30.217 & $\begin{array}{c}31.29 \\
8\end{array}$ \\
\hline & $\begin{array}{c}\text { Ciela } \\
\text { b }\end{array}$ & 4.788 & 3.932 & 3.912 & 3.872 & 3.883 & 3.899 & 3.190 & 4.071 & 3.936 & 3.874 & 3.879 & 3.190 \\
\hline & SSIM & 0.690 & 0.867 & 0.867 & 0.855 & 0.855 & 0.838 & 0.806 & 0.855 & 0.849 & 0.858 & 0.849 & 0.867 \\
\hline & HVS & 24.051 & 23.775 & $\begin{array}{c}24.23 \\
8\end{array}$ & $\begin{array}{c}24.03 \\
6\end{array}$ & $\begin{array}{c}24.06 \\
6\end{array}$ & $\begin{array}{c}24.20 \\
1\end{array}$ & 28.526 & $\begin{array}{c}24.17 \\
5\end{array}$ & $\begin{array}{c}23.97 \\
6\end{array}$ & $\begin{array}{c}24.25 \\
8\end{array}$ & 24.241 & $\begin{array}{c}28.52 \\
6\end{array}$ \\
\hline & $\begin{array}{c}\text { HVS } \\
\text { m }\end{array}$ & 25.663 & 25.177 & $\begin{array}{c}25.64 \\
3\end{array}$ & $\begin{array}{c}25.60 \\
3\end{array}$ & $\begin{array}{c}25.59 \\
9\end{array}$ & $\begin{array}{c}25.60 \\
9\end{array}$ & 30.328 & $\begin{array}{c}25.60 \\
9\end{array}$ & $\begin{array}{c}25.51 \\
4\end{array}$ & $\begin{array}{c}25.66 \\
8\end{array}$ & 25.688 & $\begin{array}{c}30.32 \\
8\end{array}$ \\
\hline \multirow[t]{5}{*}{ Img11 } & PSNR & 29.048 & 32.024 & $\begin{array}{c}32.24 \\
5\end{array}$ & $\begin{array}{c}31.67 \\
9\end{array}$ & $\begin{array}{c}31.69 \\
9\end{array}$ & $\begin{array}{c}32.22 \\
2 \\
\end{array}$ & 31.708 & $\begin{array}{c}31.77 \\
9\end{array}$ & $\begin{array}{c}31.55 \\
7 \\
\end{array}$ & $\begin{array}{c}32.24 \\
4\end{array}$ & 31.757 & $\begin{array}{c}32.24 \\
5\end{array}$ \\
\hline & $\begin{array}{c}\text { Ciela } \\
\text { b }\end{array}$ & 4.268 & 3.513 & 3.500 & 3.617 & 3.577 & 3.476 & 3.394 & 3.603 & 3.611 & 3.473 & 3.560 & 3.394 \\
\hline & SSIM & 0.726 & 0.886 & 0.887 & 0.870 & 0.878 & 0.883 & 0.843 & 0.877 & 0.873 & 0.884 & 0.867 & 0.887 \\
\hline & HVS & 26.669 & 26.089 & $\begin{array}{c}26.90 \\
7 \\
\end{array}$ & $\begin{array}{c}26.81 \\
7 \\
\end{array}$ & $\begin{array}{c}26.90 \\
5\end{array}$ & $\begin{array}{c}26.87 \\
5 \\
\end{array}$ & 28.814 & $\begin{array}{c}26.88 \\
4\end{array}$ & $\begin{array}{c}26.88 \\
7 \\
\end{array}$ & $\begin{array}{c}26.88 \\
2 \\
\end{array}$ & 26.905 & $\begin{array}{c}28.81 \\
4\end{array}$ \\
\hline & $\begin{array}{c}\text { HVS } \\
\text { m }\end{array}$ & 28.329 & 27.577 & $\begin{array}{c}28.41 \\
4\end{array}$ & $\begin{array}{c}28.43 \\
6\end{array}$ & $\begin{array}{c}28.48 \\
6\end{array}$ & $\begin{array}{c}28.36 \\
8 \\
\end{array}$ & 30.422 & $\begin{array}{c}28.43 \\
5\end{array}$ & $\begin{array}{c}28.47 \\
7 \\
\end{array}$ & $\begin{array}{c}28.37 \\
4\end{array}$ & 28.416 & $\begin{array}{c}30.42 \\
2\end{array}$ \\
\hline \multirow[t]{5}{*}{ Img12 } & PSNR & 25.869 & 28.419 & $\begin{array}{c}29.10 \\
6 \\
\end{array}$ & $\begin{array}{c}28.71 \\
5 \\
\end{array}$ & $\begin{array}{c}28.70 \\
3 \\
\end{array}$ & $\begin{array}{c}28.86 \\
9 \\
\end{array}$ & 29.150 & $\begin{array}{c}28.73 \\
2 \\
\end{array}$ & $\begin{array}{c}28.62 \\
0 \\
\end{array}$ & $\begin{array}{c}28.85 \\
5 \\
\end{array}$ & 28.744 & $\begin{array}{c}29.15 \\
0 \\
\end{array}$ \\
\hline & $\begin{array}{c}\text { Ciela } \\
\text { b }\end{array}$ & 4.506 & 3.609 & 3.494 & 3.555 & 3.569 & 3.662 & 3.149 & 3.669 & 3.613 & 3.660 & 3.558 & 3.149 \\
\hline & SSIM & 0.773 & 0.915 & 0.917 & 0.908 & 0.907 & 0.891 & 0.888 & 0.895 & 0.893 & 0.892 & 0.908 & 0.917 \\
\hline & HVS & 24.036 & 22.977 & $\begin{array}{c}24.30 \\
1\end{array}$ & $\begin{array}{c}24.32 \\
6\end{array}$ & $\begin{array}{c}24.34 \\
2\end{array}$ & $\begin{array}{c}24.14 \\
5\end{array}$ & 27.476 & $\begin{array}{c}24.24 \\
4\end{array}$ & $\begin{array}{c}24.28 \\
5\end{array}$ & $\begin{array}{c}24.15 \\
0\end{array}$ & 24.266 & $\begin{array}{c}27.47 \\
6\end{array}$ \\
\hline & $\begin{array}{c}\mathrm{HVS} \\
\mathrm{m}\end{array}$ & 25.658 & 24.416 & $\begin{array}{c}25.77 \\
6\end{array}$ & $\begin{array}{c}25.84 \\
8\end{array}$ & $\begin{array}{c}25.87 \\
8\end{array}$ & $\begin{array}{c}25.57 \\
9\end{array}$ & 29.381 & $\begin{array}{c}25.75 \\
0\end{array}$ & $\begin{array}{c}25.81 \\
7\end{array}$ & $\begin{array}{c}25.58 \\
4\end{array}$ & 25.732 & $\begin{array}{c}29.38 \\
1\end{array}$ \\
\hline \multirow[t]{5}{*}{$\begin{array}{c}\text { Averag } \\
\mathrm{e}\end{array}$} & PSNR & 28.361 & 31.578 & $\begin{array}{c}31.75 \\
8 \\
\end{array}$ & $\begin{array}{c}31.23 \\
4\end{array}$ & $\begin{array}{c}31.03 \\
8 \\
\end{array}$ & $\begin{array}{c}31.64 \\
6\end{array}$ & 31.802 & $\begin{array}{c}31.23 \\
0\end{array}$ & $\begin{array}{c}30.87 \\
9 \\
\end{array}$ & $\begin{array}{c}31.71 \\
4\end{array}$ & 31.390 & $\begin{array}{c}31.80 \\
2 \\
\end{array}$ \\
\hline & $\begin{array}{c}\text { Ciela } \\
\text { b }\end{array}$ & 4.308 & 3.441 & 3.449 & 3.440 & 3.507 & 3.459 & 3.068 & 3.537 & 3.561 & 3.429 & 3.465 & 3.068 \\
\hline & SSIM & 0.711 & 0.870 & 0.865 & 0.856 & 0.857 & 0.851 & 0.827 & 0.852 & 0.846 & 0.855 & 0.851 & 0.870 \\
\hline & HVS & 25.410 & 25.138 & $\begin{array}{c}25.72 \\
5\end{array}$ & $\begin{array}{c}25.57 \\
3 \\
\end{array}$ & $\begin{array}{c}25.63 \\
1 \\
\end{array}$ & $\begin{array}{c}25.76 \\
9\end{array}$ & 28.735 & $\begin{array}{c}25.66 \\
3 \\
\end{array}$ & $\begin{array}{c}25.57 \\
8 \\
\end{array}$ & $\begin{array}{c}25.69 \\
7 \\
\end{array}$ & 25.705 & $\begin{array}{c}28.73 \\
5\end{array}$ \\
\hline & $\begin{array}{c}\text { HVS } \\
\text { m }\end{array}$ & 27.050 & 26.556 & $\begin{array}{c}27.17 \\
4 \\
\end{array}$ & $\begin{array}{c}27.11 \\
6\end{array}$ & $\begin{array}{c}27.17 \\
7 \\
\end{array}$ & $\begin{array}{c}27.21 \\
5 \\
\end{array}$ & 30.463 & $\begin{array}{c}27.15 \\
1 \\
\end{array}$ & $\begin{array}{c}27.13 \\
0 \\
\end{array}$ & $\begin{array}{c}27.13 \\
2 \\
\end{array}$ & 27.162 & $\begin{array}{c}30.46 \\
3 \\
\end{array}$ \\
\hline
\end{tabular}

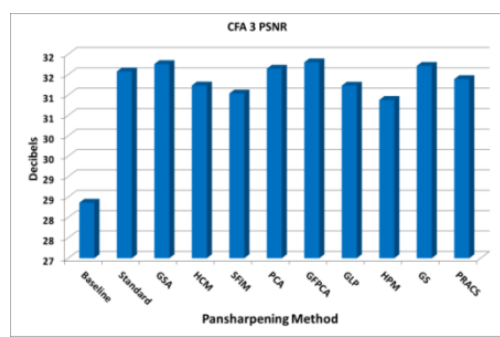

(a) PSNR

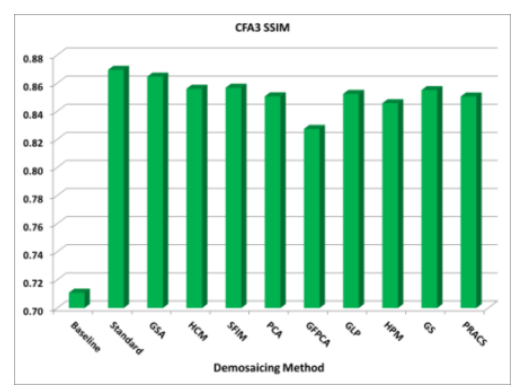

(c) SSIM

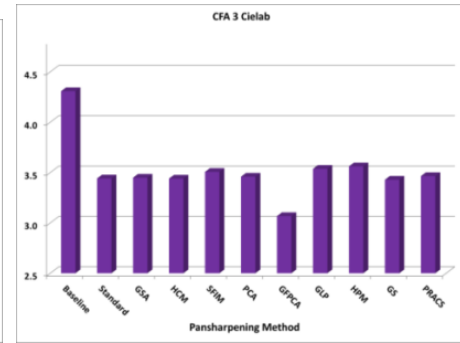

(b) CIELAB

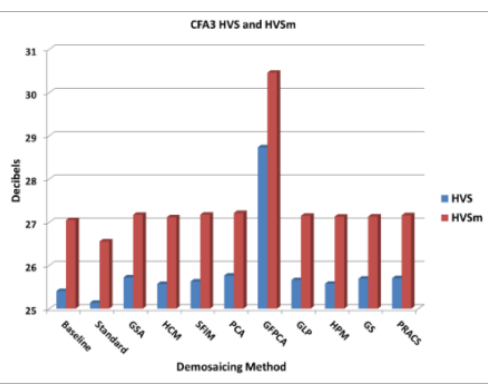

(d) HVS and HVSm

Figure 9. Averaged performance metrics of all the demosaicing results for the FOE inpainting case. 
Signal \& Image Processing: An International Journal (SIPIJ) Vol.11, No.6, December 2020

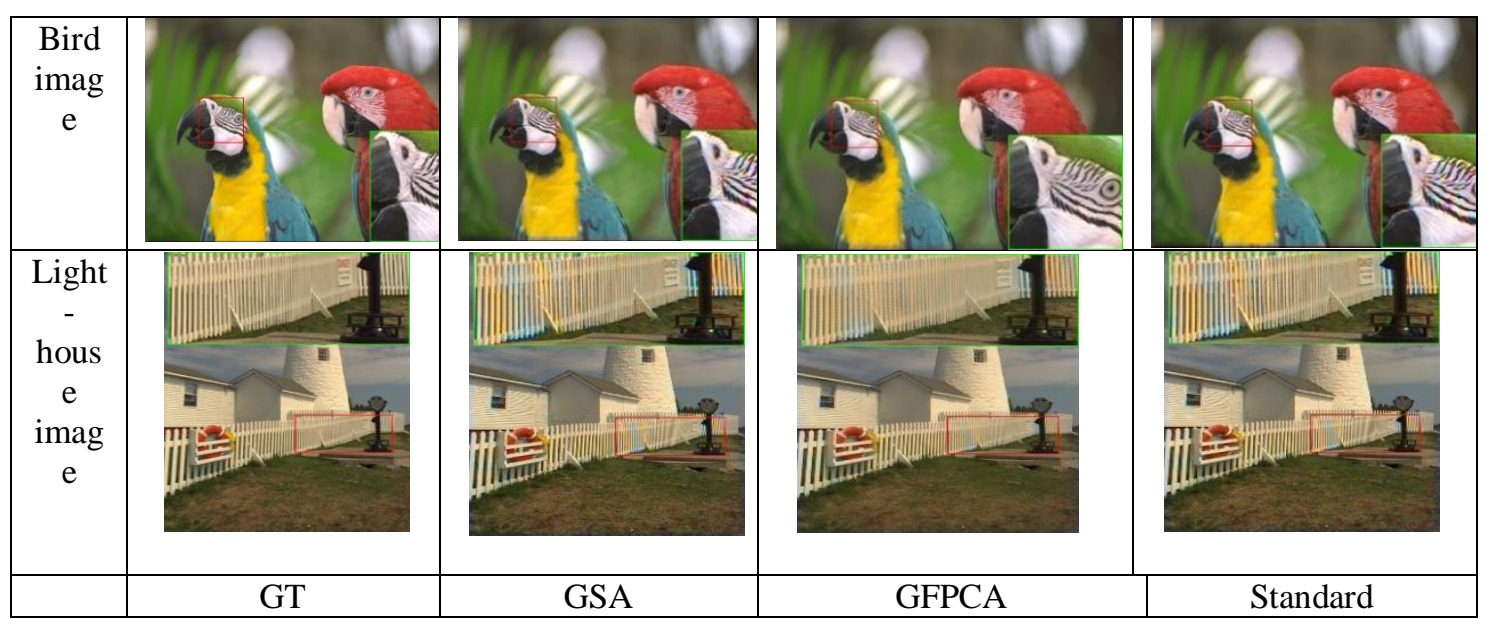

Figure 10. The ground truth image and three selected demosaiced images using the FOE inpainting method for pan band.

\subsubsection{REF + Pansharpening}

It will be important to show the best achievable demosaicing performance of those 12 Kodak images using CFA 3.0. The gap between this ideal case and what we have so far will indicate the room for further improvement. To generate the ideal demosaicing results, we used the ground truth pan images, which are generated by taking the average of the RGB bands in the original clean Kodak images. Table 4 summarizes the performance metrics for all the demosaicing results. Figure 11 plots the averaged metrics. The GFPCA has the best metrics in CIELAB, HVS, and HVSm. GSA has the best performance in terms of PSNR and the Standard method achieved the best in SSIM. From Figure 12, it is quite obvious that GFPCA has the least artifacts.

Table 4.Demosaicing results of Kodak images. Ground truth pan images were used in the pansharpening process.

\begin{tabular}{|c|c|c|c|c|c|c|c|c|c|c|c|c|c|}
\hline Image & & $\begin{array}{c}\text { Baselin } \\
\mathbf{e}\end{array}$ & $\underset{d}{\text { Standar }}$ & GSA & HCM & $\begin{array}{c}\text { SFI } \\
\text { M }\end{array}$ & PCA & $\begin{array}{c}\text { GFPC } \\
\text { A }\end{array}$ & GLP & HPM & GS & $\begin{array}{c}\text { PRAC } \\
\text { S }\end{array}$ & $\begin{array}{c}\text { Best } \\
\text { Score }\end{array}$ \\
\hline \multirow[t]{5}{*}{ Img1 } & $\begin{array}{c}\text { PSN } \\
\text { R }\end{array}$ & 32.056 & 34.252 & $\begin{array}{c}34.53 \\
9\end{array}$ & $\begin{array}{c}33.87 \\
8\end{array}$ & $\begin{array}{c}33.38 \\
5\end{array}$ & $\begin{array}{c}34.27 \\
3\end{array}$ & 33.601 & $\begin{array}{c}34.11 \\
9\end{array}$ & $\begin{array}{c}33.21 \\
8\end{array}$ & $\begin{array}{c}34.59 \\
7\end{array}$ & 33.816 & 34.597 \\
\hline & $\begin{array}{c}\text { Ciela } \\
\text { b }\end{array}$ & 2.635 & 2.261 & 2.311 & 2.382 & 2.635 & 2.333 & 2.688 & 2.340 & 2.673 & 2.236 & 2.376 & 2.236 \\
\hline & SSIM & 0.746 & 0.896 & 0.888 & 0.866 & 0.873 & 0.885 & 0.837 & 0.891 & 0.869 & 0.896 & 0.848 & 0.896 \\
\hline & HVS & 28.179 & 27.292 & $\begin{array}{c}28.31 \\
3\end{array}$ & $\begin{array}{c}28.13 \\
0\end{array}$ & $\begin{array}{c}28.26 \\
0\end{array}$ & $\begin{array}{c}28.36 \\
3\end{array}$ & 28.112 & $\begin{array}{c}28.14 \\
5\end{array}$ & $\begin{array}{c}28.23 \\
2\end{array}$ & $\begin{array}{c}28.29 \\
1\end{array}$ & 28.332 & 28.363 \\
\hline & $\begin{array}{c}\mathrm{HVS} \\
\mathrm{m}\end{array}$ & 29.715 & 28.701 & $\begin{array}{c}29.73 \\
0 \\
\end{array}$ & $\begin{array}{c}29.64 \\
2 \\
\end{array}$ & $\begin{array}{c}29.76 \\
2 \\
\end{array}$ & $\begin{array}{c}29.78 \\
9\end{array}$ & 28.899 & $\begin{array}{c}29.61 \\
0 \\
\end{array}$ & $\begin{array}{c}29.74 \\
3 \\
\end{array}$ & $\begin{array}{c}29.69 \\
8 \\
\end{array}$ & 29.771 & 29.789 \\
\hline \multirow[t]{5}{*}{ Img2 } & $\begin{array}{c}\text { PSN } \\
\text { R }\end{array}$ & 26.887 & 31.613 & $\begin{array}{c}31.55 \\
1\end{array}$ & $\begin{array}{c}31.05 \\
3\end{array}$ & $\begin{array}{c}30.66 \\
9\end{array}$ & $\begin{array}{c}31.43 \\
2 \\
\end{array}$ & 30.835 & $\begin{array}{c}30.62 \\
4 \\
\end{array}$ & $\begin{array}{c}30.33 \\
9\end{array}$ & $\begin{array}{c}31.56 \\
1\end{array}$ & 31.213 & 31.613 \\
\hline & $\begin{array}{c}\text { Ciela } \\
\text { b }\end{array}$ & 4.810 & 3.436 & 3.499 & 3.548 & 3.608 & 3.568 & 3.040 & 3.691 & 3.684 & 3.501 & 3.594 & 3.040 \\
\hline & SSIM & 0.695 & 0.906 & 0.906 & 0.893 & 0.892 & 0.881 & 0.851 & 0.890 & 0.886 & 0.886 & 0.890 & 0.906 \\
\hline & HVS & 23.982 & 24.346 & $\begin{array}{c}24.46 \\
4\end{array}$ & $\begin{array}{c}24.26 \\
9\end{array}$ & $\begin{array}{c}24.14 \\
6\end{array}$ & $\begin{array}{c}24.72 \\
3\end{array}$ & 28.156 & $\begin{array}{c}24.22 \\
5\end{array}$ & $\begin{array}{c}23.98 \\
9\end{array}$ & $\begin{array}{c}24.38 \\
7\end{array}$ & 24.496 & 28.156 \\
\hline & $\begin{array}{c}\mathrm{HVS} \\
\mathrm{m}\end{array}$ & 25.505 & 25.515 & $\begin{array}{c}25.67 \\
9\end{array}$ & $\begin{array}{c}25.54 \\
1\end{array}$ & $\begin{array}{c}25.45 \\
8\end{array}$ & $\begin{array}{c}25.94 \\
7\end{array}$ & 30.070 & $\begin{array}{c}25.48 \\
6\end{array}$ & $\begin{array}{c}25.33 \\
1\end{array}$ & $\begin{array}{c}25.57 \\
8\end{array}$ & 25.692 & 30.070 \\
\hline \multirow[t]{5}{*}{ Img3 } & $\begin{array}{c}\text { PSN } \\
\text { R }\end{array}$ & 30.887 & 33.356 & $\begin{array}{c}33.25 \\
5\end{array}$ & $\begin{array}{c}32.33 \\
3\end{array}$ & $\begin{array}{c}32.62 \\
1\end{array}$ & $\begin{array}{c}33.40 \\
7\end{array}$ & 34.886 & $\begin{array}{c}32.95 \\
1\end{array}$ & $\begin{array}{c}32.47 \\
6\end{array}$ & $\begin{array}{c}33.45 \\
1\end{array}$ & 32.908 & 34.886 \\
\hline & $\begin{array}{c}\text { Ciela } \\
\mathrm{b}\end{array}$ & 3.734 & 3.248 & 3.236 & 3.412 & 3.341 & 3.161 & 2.819 & 3.217 & 3.384 & 3.141 & 3.308 & 2.819 \\
\hline & SSIM & 0.792 & 0.920 & 0.913 & 0.900 & 0.910 & 0.911 & 0.899 & 0.910 & 0.904 & 0.911 & 0.898 & 0.920 \\
\hline & HVS & 26.958 & 26.684 & $\begin{array}{c}26.88 \\
0\end{array}$ & $\begin{array}{c}26.62 \\
2\end{array}$ & $\begin{array}{c}26.74 \\
6\end{array}$ & $\begin{array}{c}27.05 \\
8\end{array}$ & 30.303 & $\begin{array}{c}26.77 \\
3\end{array}$ & $\begin{array}{c}26.69 \\
7\end{array}$ & $\begin{array}{c}27.02 \\
5\end{array}$ & 26.971 & 30.303 \\
\hline & $\begin{array}{c}\mathrm{HVS} \\
\mathrm{m}\end{array}$ & 28.736 & 28.311 & $\begin{array}{c}28.53 \\
9\end{array}$ & $\begin{array}{c}28.44 \\
9\end{array}$ & $\begin{array}{c}28.50 \\
6\end{array}$ & $\begin{array}{c}28.71 \\
1\end{array}$ & 31.578 & $\begin{array}{c}28.45 \\
9\end{array}$ & $\begin{array}{c}28.46 \\
6\end{array}$ & $\begin{array}{c}28.67 \\
3\end{array}$ & 28.641 & 31.578 \\
\hline $\operatorname{Img} 4$ & $\begin{array}{c}\text { PSN } \\
\text { R }\end{array}$ & 22.850 & 27.850 & $\begin{array}{c}28.54 \\
7\end{array}$ & $\begin{array}{c}28.01 \\
1\end{array}$ & $\begin{array}{c}27.60 \\
9\end{array}$ & $\begin{array}{c}27.71 \\
8\end{array}$ & 27.470 & $\begin{array}{c}27.37 \\
8\end{array}$ & $\begin{array}{c}27.36 \\
8\end{array}$ & $\begin{array}{c}27.70 \\
9\end{array}$ & 27.906 & 28.547 \\
\hline
\end{tabular}


Signal \& Image Processing: An International Journal (SIPIJ) Vol.11, No.6, December 2020

\begin{tabular}{|c|c|c|c|c|c|c|c|c|c|c|c|c|c|}
\hline & $\begin{array}{c}\text { Ciela } \\
\text { b }\end{array}$ & 7.419 & 5.037 & 4.972 & 4.729 & 4.929 & 5.327 & 4.629 & 5.412 & 5.052 & 5.306 & 4.978 & 4.629 \\
\hline & SSIM & 0.758 & 0.953 & 0.952 & 0.946 & 0.942 & 0.927 & 0.913 & 0.939 & 0.936 & 0.929 & 0.940 & 0.953 \\
\hline & HVS & 20.336 & 20.427 & $\begin{array}{c}21.25 \\
3\end{array}$ & $\begin{array}{c}21.21 \\
8\end{array}$ & $\begin{array}{c}21.30 \\
6\end{array}$ & $\begin{array}{c}21.13 \\
3\end{array}$ & 24.531 & $\begin{array}{c}21.23 \\
1\end{array}$ & $\begin{array}{c}21.30 \\
7\end{array}$ & $\begin{array}{c}21.01 \\
1\end{array}$ & 21.081 & 24.531 \\
\hline & $\begin{array}{c}\mathrm{HVS} \\
\mathrm{m}\end{array}$ & 22.007 & 21.670 & $\begin{array}{c}22.55 \\
4\end{array}$ & $\begin{array}{c}22.60 \\
6\end{array}$ & $\begin{array}{c}22.70 \\
7\end{array}$ & $\begin{array}{c}22.41 \\
8\end{array}$ & 26.453 & $\begin{array}{c}22.60 \\
3\end{array}$ & $\begin{array}{c}22.73 \\
3\end{array}$ & $\begin{array}{c}22.28 \\
1\end{array}$ & 22.401 & 26.453 \\
\hline \multirow[t]{5}{*}{ Img5 } & $\begin{array}{c}\text { PSN } \\
\mathrm{R} \\
\end{array}$ & 30.918 & 34.782 & $\begin{array}{c}34.33 \\
7\end{array}$ & $\begin{array}{c}34.28 \\
5\end{array}$ & $\begin{array}{c}34.07 \\
2\end{array}$ & $\begin{array}{c}34.51 \\
4\end{array}$ & 35.281 & $\begin{array}{c}34.12 \\
6\end{array}$ & $\begin{array}{c}33.83 \\
3\end{array}$ & $\begin{array}{c}34.87 \\
4\end{array}$ & 34.328 & 35.281 \\
\hline & $\begin{array}{c}\text { Ciela } \\
\text { b }\end{array}$ & 2.539 & 1.955 & 2.146 & 1.903 & 1.990 & 2.033 & 1.817 & 2.034 & 2.031 & 1.917 & 2.049 & 1.817 \\
\hline & SSIM & 0.677 & 0.928 & 0.903 & 0.917 & 0.917 & 0.907 & 0.831 & 0.914 & 0.910 & 0.920 & 0.890 & 0.928 \\
\hline & HVS & 27.739 & 27.778 & $\begin{array}{c}28.16 \\
3\end{array}$ & $\begin{array}{c}28.14 \\
4\end{array}$ & $\begin{array}{c}28.07 \\
1\end{array}$ & $\begin{array}{c}28.42 \\
8\end{array}$ & 30.870 & $\begin{array}{c}28.17 \\
1\end{array}$ & $\begin{array}{c}28.02 \\
1\end{array}$ & $\begin{array}{c}28.21 \\
3\end{array}$ & 28.219 & 30.870 \\
\hline & $\begin{array}{c}\text { HVS } \\
\mathrm{m}\end{array}$ & 29.436 & 29.212 & $\begin{array}{c}29.63 \\
2\end{array}$ & $\begin{array}{c}29.66 \\
7 \\
\end{array}$ & $\begin{array}{c}29.69 \\
8\end{array}$ & $\begin{array}{c}29.90 \\
9 \\
\end{array}$ & 32.173 & $\begin{array}{c}29.70 \\
5\end{array}$ & $\begin{array}{c}29.67 \\
1\end{array}$ & $\begin{array}{c}29.66 \\
0 \\
\end{array}$ & 29.679 & 32.173 \\
\hline \multirow[t]{5}{*}{ Img6 } & $\begin{array}{c}\text { PSN } \\
\text { R }\end{array}$ & 27.796 & 31.616 & $\begin{array}{c}31.77 \\
5 \\
\end{array}$ & $\begin{array}{c}30.99 \\
7 \\
\end{array}$ & $\begin{array}{c}30.93 \\
7 \\
\end{array}$ & $\begin{array}{c}31.81 \\
6 \\
\end{array}$ & 32.177 & $\begin{array}{c}31.21 \\
3 \\
\end{array}$ & $\begin{array}{c}30.64 \\
6\end{array}$ & $\begin{array}{c}31.74 \\
0 \\
\end{array}$ & 31.315 & 32.177 \\
\hline & $\begin{array}{c}\text { Ciela } \\
b\end{array}$ & 5.508 & 4.331 & 4.611 & 4.230 & 4.197 & 4.348 & 3.619 & 4.398 & 4.339 & 4.385 & 4.341 & 3.619 \\
\hline & SSIM & 0.720 & 0.934 & 0.909 & 0.913 & 0.916 & 0.902 & 0.874 & 0.916 & 0.904 & 0.898 & 0.902 & 0.934 \\
\hline & HVS & 24.627 & 24.620 & $\begin{array}{c}25.06 \\
8\end{array}$ & $\begin{array}{c}24.73 \\
9\end{array}$ & $\begin{array}{c}24.90 \\
3\end{array}$ & $\begin{array}{c}25.00 \\
2\end{array}$ & 28.061 & $\begin{array}{c}25.07 \\
0\end{array}$ & $\begin{array}{c}24.86 \\
8\end{array}$ & $\begin{array}{c}25.05 \\
7\end{array}$ & 24.969 & 28.061 \\
\hline & $\begin{array}{c}\text { HVS } \\
\text { m }\end{array}$ & 26.295 & 26.029 & $\begin{array}{c}26.48 \\
4\end{array}$ & $\begin{array}{c}26.31 \\
1\end{array}$ & $\begin{array}{c}26.42 \\
2\end{array}$ & $\begin{array}{c}26.41 \\
3\end{array}$ & 29.485 & $\begin{array}{c}26.51 \\
2\end{array}$ & $\begin{array}{c}26.39 \\
5\end{array}$ & $\begin{array}{c}26.47 \\
4\end{array}$ & 26.433 & 29.485 \\
\hline \multirow[t]{5}{*}{ Img7 } & $\begin{array}{c}\text { PSN } \\
\text { R }\end{array}$ & 30.561 & 35.654 & $\begin{array}{c}35.51 \\
6\end{array}$ & $\begin{array}{c}34.95 \\
4\end{array}$ & $\begin{array}{c}34.41 \\
0\end{array}$ & $\begin{array}{c}35.47 \\
9\end{array}$ & 34.405 & $\begin{array}{c}34.45 \\
3\end{array}$ & $\begin{array}{c}34.06 \\
9\end{array}$ & $\begin{array}{c}35.51 \\
1 \\
\end{array}$ & 35.273 & 35.654 \\
\hline & $\begin{array}{c}\text { Ciela } \\
\text { b }\end{array}$ & 3.598 & 2.496 & 2.535 & 2.554 & 2.633 & 2.540 & 2.374 & 2.696 & 2.694 & 2.524 & 2.599 & 2.374 \\
\hline & SSIM & 0.741 & 0.942 & 0.940 & 0.933 & 0.930 & 0.936 & 0.882 & 0.928 & 0.924 & 0.936 & 0.929 & 0.942 \\
\hline & HVS & 27.968 & 28.420 & $\begin{array}{c}28.42 \\
0\end{array}$ & $\begin{array}{c}28.24 \\
5\end{array}$ & $\begin{array}{c}28.06 \\
9\end{array}$ & $\begin{array}{c}28.52 \\
8\end{array}$ & 32.628 & $\begin{array}{c}28.19 \\
5\end{array}$ & $\begin{array}{c}27.88 \\
2\end{array}$ & $\begin{array}{c}28.44 \\
0\end{array}$ & 28.500 & 32.628 \\
\hline & $\begin{array}{c}\text { HVS } \\
\mathrm{m}\end{array}$ & 29.520 & 29.564 & $\begin{array}{c}29.56 \\
6\end{array}$ & $\begin{array}{c}29.48 \\
8 \\
\end{array}$ & $\begin{array}{c}29.34 \\
5\end{array}$ & $\begin{array}{c}29.68 \\
0 \\
\end{array}$ & 34.727 & $\begin{array}{c}29.40 \\
2 \\
\end{array}$ & $\begin{array}{c}29.17 \\
6\end{array}$ & $\begin{array}{c}29.58 \\
8 \\
\end{array}$ & 29.663 & 34.727 \\
\hline \multirow[t]{5}{*}{ Img8 } & $\begin{array}{c}\text { PSN } \\
\text { R }\end{array}$ & 27.013 & 31.282 & $\begin{array}{c}31.71 \\
7\end{array}$ & $\begin{array}{c}30.88 \\
0\end{array}$ & $\begin{array}{c}30.67 \\
7\end{array}$ & $\begin{array}{c}31.22 \\
1\end{array}$ & 30.804 & $\begin{array}{c}30.76 \\
2\end{array}$ & $\begin{array}{c}30.41 \\
9\end{array}$ & $\begin{array}{c}31.33 \\
1\end{array}$ & 31.015 & 31.717 \\
\hline & $\begin{array}{c}\text { Ciela } \\
\text { b }\end{array}$ & 4.658 & 3.468 & 3.310 & 3.536 & 3.476 & 3.503 & 3.051 & 3.546 & 3.534 & 3.449 & 3.596 & 3.051 \\
\hline & SSIM & 0.740 & 0.937 & 0.937 & 0.921 & 0.926 & 0.923 & 0.890 & 0.927 & 0.921 & 0.927 & 0.913 & 0.937 \\
\hline & HVS & 24.453 & 23.969 & $\begin{array}{c}25.01 \\
5\end{array}$ & $\begin{array}{c}24.81 \\
1\end{array}$ & $\begin{array}{c}24.91 \\
6\end{array}$ & $\begin{array}{c}25.06 \\
0\end{array}$ & 29.237 & $\begin{array}{c}24.95 \\
5\end{array}$ & $\begin{array}{c}24.90 \\
7\end{array}$ & $\begin{array}{c}24.88 \\
9\end{array}$ & 24.909 & 29.237 \\
\hline & $\begin{array}{c}\text { HVS } \\
\mathrm{m}\end{array}$ & 26.129 & 25.317 & $\begin{array}{c}26.42 \\
0\end{array}$ & $\begin{array}{c}26.32 \\
6\end{array}$ & $\begin{array}{c}26.41 \\
6\end{array}$ & $\begin{array}{c}26.45 \\
5\end{array}$ & 31.046 & $\begin{array}{c}26.41 \\
5\end{array}$ & $\begin{array}{c}26.42 \\
5\end{array}$ & $\begin{array}{c}26.26 \\
4\end{array}$ & 26.311 & 31.046 \\
\hline \multirow[t]{5}{*}{ Img9 } & $\begin{array}{c}\text { PSN } \\
\text { R }\end{array}$ & 29.869 & 32.768 & $\begin{array}{c}33.33 \\
8\end{array}$ & $\begin{array}{c}32.63 \\
6\end{array}$ & $\begin{array}{c}32.07 \\
8\end{array}$ & $\begin{array}{c}33.41 \\
4\end{array}$ & 34.743 & $\begin{array}{c}32.82 \\
3\end{array}$ & $\begin{array}{c}31.91 \\
2\end{array}$ & $\begin{array}{c}33.40 \\
7\end{array}$ & 32.908 & 34.743 \\
\hline & $\begin{array}{c}\text { Ciela } \\
\text { b }\end{array}$ & 3.037 & 2.555 & 2.462 & 2.437 & 2.811 & 2.371 & 2.078 & 2.539 & 2.892 & 2.371 & 2.457 & 2.078 \\
\hline & SSIM & 0.512 & 0.665 & 0.668 & 0.653 & 0.653 & 0.618 & 0.636 & 0.609 & 0.594 & 0.619 & 0.651 & 0.668 \\
\hline & HVS & 26.315 & 25.823 & $\begin{array}{c}26.77 \\
0\end{array}$ & $\begin{array}{c}26.60 \\
7\end{array}$ & $\begin{array}{c}26.78 \\
7\end{array}$ & $\begin{array}{c}26.78 \\
1\end{array}$ & 30.702 & $\begin{array}{c}26.83 \\
3\end{array}$ & $\begin{array}{c}26.76 \\
8\end{array}$ & $\begin{array}{c}26.81 \\
4\end{array}$ & 26.668 & 30.702 \\
\hline & $\begin{array}{c}\text { HVS } \\
\mathrm{m}\end{array}$ & 27.938 & 27.232 & $\begin{array}{c}28.18 \\
1\end{array}$ & $\begin{array}{c}28.12 \\
1\end{array}$ & $\begin{array}{c}28.27 \\
7\end{array}$ & $\begin{array}{c}28.19 \\
2\end{array}$ & 32.204 & $\begin{array}{c}28.28 \\
5\end{array}$ & $\begin{array}{c}28.26 \\
5\end{array}$ & $\begin{array}{c}28.22 \\
9\end{array}$ & 28.097 & 32.204 \\
\hline \multirow[t]{5}{*}{$\operatorname{Img} 10$} & $\begin{array}{c}\text { PSN } \\
\text { R }\end{array}$ & 27.156 & 31.142 & $\begin{array}{c}31.37 \\
3\end{array}$ & $\begin{array}{c}30.60 \\
0\end{array}$ & $\begin{array}{c}30.49 \\
1\end{array}$ & $\begin{array}{c}31.21 \\
2\end{array}$ & 32.149 & $\begin{array}{c}30.53 \\
8\end{array}$ & $\begin{array}{c}30.20 \\
9\end{array}$ & $\begin{array}{c}31.16 \\
1\end{array}$ & 30.910 & 32.149 \\
\hline & $\begin{array}{c}\text { Ciela } \\
\text { b }\end{array}$ & 4.755 & 3.702 & 3.688 & 3.630 & 3.639 & 3.703 & 3.025 & 3.875 & 3.717 & 3.665 & 3.671 & 3.025 \\
\hline & SSIM & 0.696 & 0.916 & 0.916 & 0.903 & 0.905 & 0.881 & 0.829 & 0.905 & 0.899 & 0.904 & 0.894 & 0.916 \\
\hline & HVS & 24.115 & 23.899 & $\begin{array}{c}24.40 \\
5\end{array}$ & $\begin{array}{c}24.19 \\
2\end{array}$ & $\begin{array}{c}24.20 \\
9\end{array}$ & $\begin{array}{c}24.36 \\
5\end{array}$ & 29.045 & $\begin{array}{c}24.32 \\
7\end{array}$ & $\begin{array}{c}24.11 \\
2\end{array}$ & $\begin{array}{c}24.42 \\
4\end{array}$ & 24.404 & 29.045 \\
\hline & $\begin{array}{c}\text { HVS } \\
\mathrm{m}\end{array}$ & 25.720 & 25.235 & $\begin{array}{c}25.73 \\
8\end{array}$ & $\begin{array}{c}25.70 \\
3 \\
\end{array}$ & $\begin{array}{c}25.67 \\
7\end{array}$ & $\begin{array}{c}25.70 \\
4\end{array}$ & 30.552 & $\begin{array}{c}25.69 \\
3\end{array}$ & $\begin{array}{c}25.59 \\
4\end{array}$ & $\begin{array}{c}25.76 \\
3 \\
\end{array}$ & 25.790 & 30.552 \\
\hline \multirow[t]{5}{*}{ Img11 } & $\begin{array}{c}\text { PSN } \\
\text { R }\end{array}$ & 29.136 & 32.760 & $\begin{array}{c}33.04 \\
3\end{array}$ & $\begin{array}{c}32.33 \\
6\end{array}$ & $\begin{array}{c}32.37 \\
0\end{array}$ & $\begin{array}{c}32.99 \\
8\end{array}$ & 32.289 & $\begin{array}{c}32.46 \\
6\end{array}$ & $\begin{array}{c}32.18 \\
1\end{array}$ & $\begin{array}{c}33.02 \\
6\end{array}$ & 32.385 & 33.043 \\
\hline & $\begin{array}{c}\text { Ciela } \\
\text { b }\end{array}$ & 4.238 & 3.313 & 3.300 & 3.430 & 3.378 & 3.278 & 3.265 & 3.424 & 3.427 & 3.276 & 3.385 & 3.265 \\
\hline & SSIM & 0.731 & 0.919 & 0.919 & 0.902 & 0.911 & 0.914 & 0.868 & 0.910 & 0.906 & 0.916 & 0.898 & 0.919 \\
\hline & HVS & 26.758 & 26.210 & $\begin{array}{c}27.10 \\
8\end{array}$ & $\begin{array}{c}26.99 \\
6\end{array}$ & $\begin{array}{c}27.08 \\
3\end{array}$ & $\begin{array}{c}27.07 \\
4\end{array}$ & 29.120 & $\begin{array}{c}27.06 \\
7\end{array}$ & $\begin{array}{c}27.05 \\
9\end{array}$ & $\begin{array}{c}27.08 \\
0\end{array}$ & 27.104 & 29.120 \\
\hline & $\begin{array}{c}\text { HVS } \\
\mathrm{m}\end{array}$ & 28.408 & 27.623 & $\begin{array}{c}28.52 \\
4\end{array}$ & $\begin{array}{c}28.53 \\
6\end{array}$ & $\begin{array}{c}28.57 \\
4\end{array}$ & $\begin{array}{c}28.47 \\
4\end{array}$ & 30.554 & $\begin{array}{c}28.53 \\
0\end{array}$ & $\begin{array}{c}28.56 \\
4\end{array}$ & $\begin{array}{c}28.47 \\
9\end{array}$ & 28.531 & 30.554 \\
\hline \multirow[t]{5}{*}{$\operatorname{Img} 12$} & $\begin{array}{c}\text { PSN } \\
\text { R }\end{array}$ & 25.977 & 29.260 & $\begin{array}{c}30.25 \\
5\end{array}$ & $\begin{array}{c}29.75 \\
8 \\
\end{array}$ & $\begin{array}{c}29.75 \\
6\end{array}$ & $\begin{array}{c}29.91 \\
5 \\
\end{array}$ & 30.136 & $\begin{array}{c}29.71 \\
1\end{array}$ & $\begin{array}{c}29.59 \\
5\end{array}$ & $\begin{array}{c}29.89 \\
4\end{array}$ & 29.716 & 30.255 \\
\hline & $\begin{array}{c}\text { Ciela } \\
\text { b }\end{array}$ & 4.472 & 3.408 & 3.272 & 3.327 & 3.348 & 3.475 & 2.983 & 3.486 & 3.409 & 3.473 & 3.344 & 2.983 \\
\hline & SSIM & 0.778 & 0.940 & 0.942 & 0.933 & 0.932 & 0.914 & 0.910 & 0.920 & 0.918 & 0.915 & 0.932 & 0.942 \\
\hline & HVS & 24.186 & 23.100 & $\begin{array}{c}24.70 \\
2 \\
\end{array}$ & $\begin{array}{c}24.75 \\
0 \\
\end{array}$ & $\begin{array}{c}24.76 \\
1 \\
\end{array}$ & $\begin{array}{c}24.52 \\
0 \\
\end{array}$ & 28.263 & $\begin{array}{c}24.63 \\
0 \\
\end{array}$ & $\begin{array}{c}24.72 \\
0 \\
\end{array}$ & $\begin{array}{c}24.52 \\
3 \\
\end{array}$ & 24.646 & 28.263 \\
\hline & HVS & 25.815 & 24.465 & 26.07 & 26.18 & 26.19 & 25.85 & 29.911 & 26.04 & 26.16 & 25.85 & 26.020 & 29.911 \\
\hline
\end{tabular}


Signal \& Image Processing: An International Journal (SIPIJ) Vol.11, No.6, December 2020

\begin{tabular}{|c|c|c|c|c|c|c|c|c|c|c|c|c|c|}
\hline & $\mathrm{m}$ & & & 4 & 4 & 5 & 2 & & 7 & 7 & 6 & & \\
\hline $\begin{array}{c}\text { Averag } \\
\text { e }\end{array}$ & $\begin{array}{c}\text { PSN } \\
\text { R }\end{array}$ & 28.426 & 32.195 & $\begin{array}{c}32.43 \\
7\end{array}$ & $\begin{array}{c}31.81 \\
0\end{array}$ & $\begin{array}{c}31.59 \\
0\end{array}$ & $\begin{array}{c}32.28 \\
3\end{array}$ & 32.398 & $\begin{array}{c}31.76 \\
4\end{array}$ & $\begin{array}{c}31.35 \\
5\end{array}$ & $\begin{array}{c}32.35 \\
5\end{array}$ & 31.974 & 32.437 \\
\hline & $\begin{array}{c}\text { Ciela } \\
\text { b }\end{array}$ & 4.283 & 3.268 & 3.278 & 3.260 & 3.332 & 3.303 & 2.949 & 3.388 & 3.403 & 3.270 & 3.308 & 2.949 \\
\hline & SSIM & 0.715 & 0.905 & 0.899 & 0.890 & 0.892 & 0.883 & 0.852 & 0.888 & 0.881 & 0.888 & 0.882 & 0.905 \\
\hline & HVS & 25.468 & 25.214 & $\begin{array}{c}25.88 \\
0\end{array}$ & $\begin{array}{c}25.72 \\
7\end{array}$ & $\begin{array}{c}25.77 \\
1\end{array}$ & $\begin{array}{c}25.92 \\
0\end{array}$ & 29.086 & $\begin{array}{c}25.80 \\
2\end{array}$ & $\begin{array}{c}25.71 \\
3\end{array}$ & $\begin{array}{c}25.84 \\
6\end{array}$ & 25.858 & 29.086 \\
\hline & $\begin{array}{c}\text { HVS } \\
\mathrm{m}\end{array}$ & 27.102 & 26.573 & $\begin{array}{c}27.26 \\
0\end{array}$ & $\begin{array}{c}27.21 \\
5\end{array}$ & $\begin{array}{c}27.25 \\
3\end{array}$ & $\begin{array}{c}27.29 \\
5\end{array}$ & 30.638 & $\begin{array}{c}27.22 \\
9\end{array}$ & $\begin{array}{c}27.21 \\
1\end{array}$ & $\begin{array}{c}27.21 \\
2\end{array}$ & 27.253 & 30.638 \\
\hline
\end{tabular}

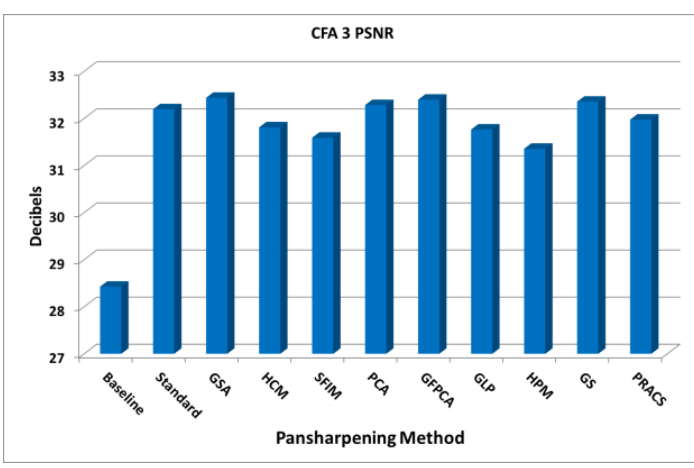

(a) PSNR

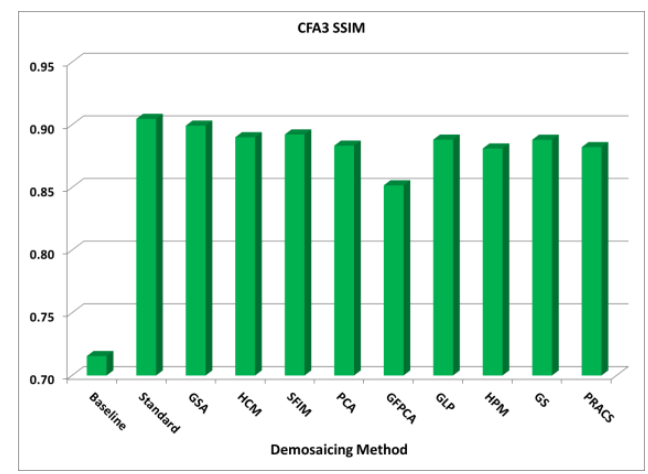

(c) SSIM

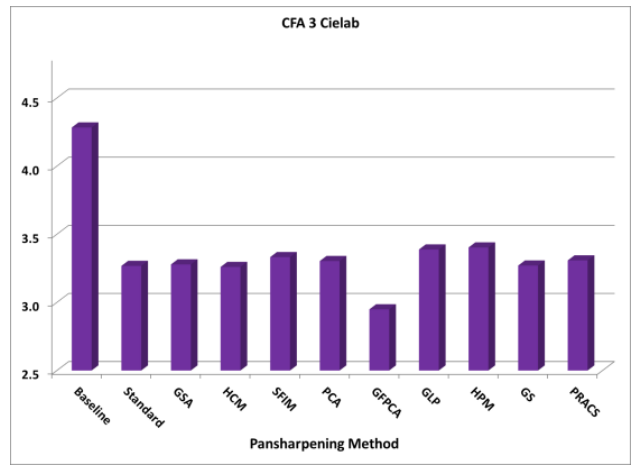

(b) CIELAB

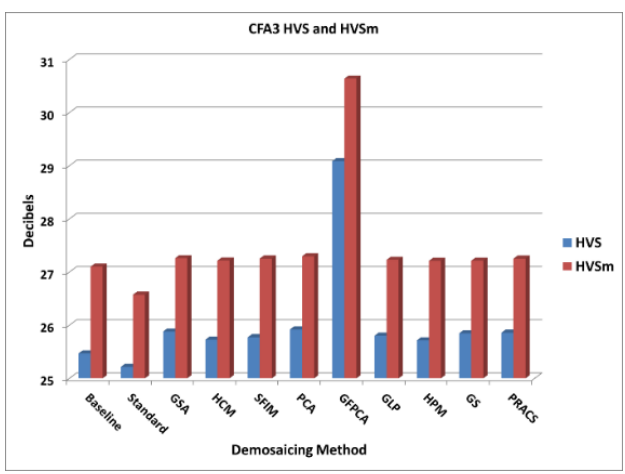

(d) HVS and HVSm

Figure 11. Averaged performance metrics of all the demosaicing results for the ground truth pan case.

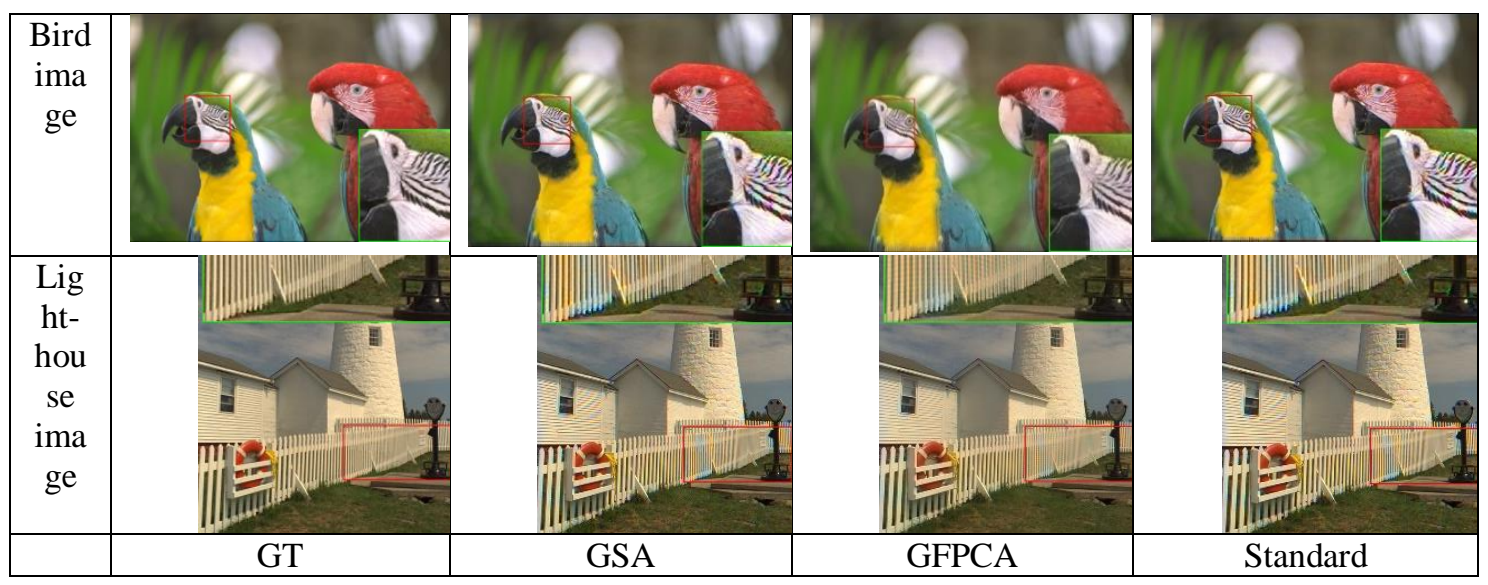

Figure 12. The ground truth image and three selected demosaiced images using the ground truth pan case. 
Signal \& Image Processing: An International Journal (SIPIJ) Vol.11, No.6, December 2020

\subsection{Discussions and Comparisons}

We summarize the key results in Tables 2 to 4 and put them into Table 5 and Figure 13. We have the following observations:

- In terms of PSNR, the combination of FOE and GFPCA improved over the combination of LDI-NAT and GSA by $0.11 \mathrm{~dB}$. There is still a somewhat big gap of $0.635 \mathrm{~dB}$ between FOE/GFPCA and GT/GSA.

- In terms of CIELAB, the difference between the FOE/GFPCA and LDI-NAT/GSA is 0.033 whereas the difference between GT/GFPCA and FOE/GFPCA is 0.119. Relatively speaking, there is still a gap for further improvement.

- In terms of SSIM, the difference between FOE/Standard and LDI-NAT/Standard is 0.003 and the difference between GT/Standard and FOE/Standard is 0.035 .

- In terms of HVS, the difference between FOE/GFPCA and LDI-NAT/GFPCA is $0.117 \mathrm{~dB}$ and the difference between GT/GFPCA and FOE/GFPCA is $0.351 \mathrm{~dB}$.

- In terms of HVSm, the difference between FOE/GFPCA and LDI-NAT/GFPCA is 0.002 $\mathrm{dB}$ and the difference between GT/GFPCA and FOE/GFPCA is $0.175 \mathrm{~dB}$.

The above observations also answer the two questions raised in Section 1. First, after some extensive experiments, it was found that there do exist better algorithms (FOE and inpaint-nans) than the LDI-NAT method. Second, we also quantify the performance gain of the better algorithms. In short, it appears that the best inpainting algorithm (FOE) closes the gap between the FOE and the ideal case. However, even the demosaicing results with GT pan may still have room for improvement, which will be a future topic to pursue.

Table 5.Demosaicing results

\begin{tabular}{|c|c|c|c|}
\hline & LDI-NAT for Pan & FOE for Pan & Reference (GT) Pan \\
\hline Metrics & Metric/ Best PS & Metric/ Best PS & Metric/Best PS \\
\hline PSNR (dB) & $31.692 /$ GSA & $31.802 /$ GFPCA & $32.437 /$ GSA \\
\hline CIELAB & $3.101 /$ GFPCA & $3.068 /$ GFPCA & $2.949 /$ GFPCA \\
\hline SSIM & $0.867 /$ Standard & $0.870 /$ Standard & $0.905 /$ Standard \\
\hline HVS $(\mathrm{dB})$ & $28.618 /$ GFPCA & $28.735 /$ GFPCA & $29.086 /$ GFPCA \\
\hline HVSm $(\mathrm{dB})$ & $30.465 / G F P C A$ & $30.463 / G F P C A$ & $30.638 / G F P C A$ \\
\hline
\end{tabular}


Signal \& Image Processing: An International Journal (SIPIJ) Vol.11, No.6, December 2020

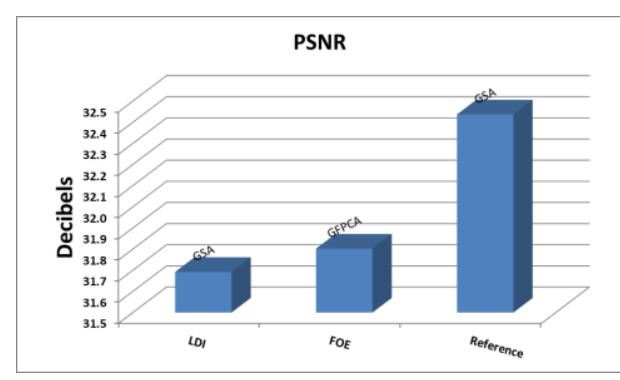

(a) PSNR

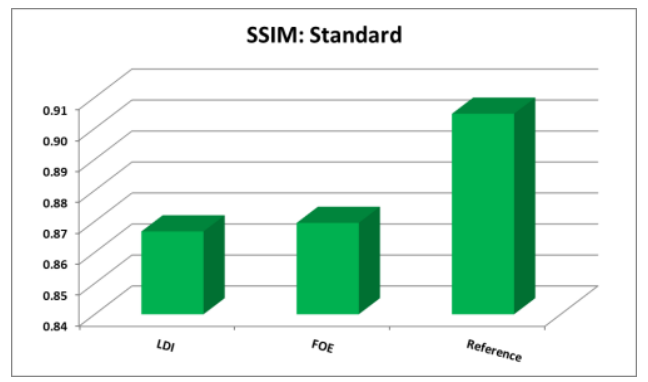

(c) SSIM

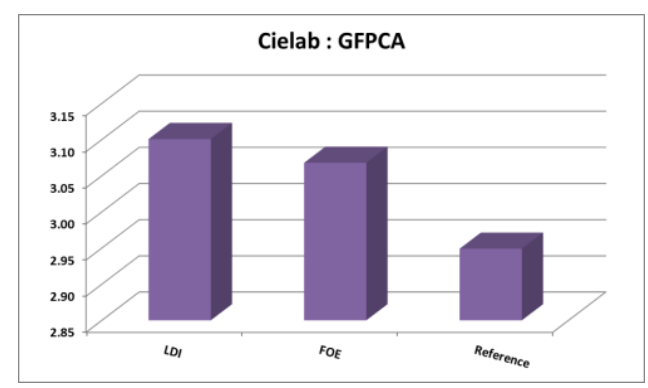

(b) CIELAB

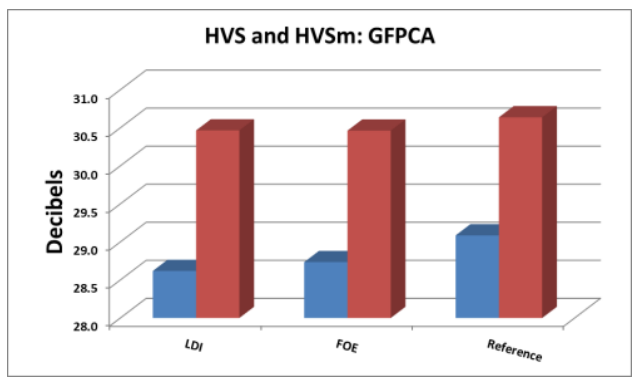

(d) HVS and HVSm

Figure 13. Comparison of the demosaicing results of using different combinations of inpainting and pansharpening algorithms.

\section{Conclusions}

In this paper, we focus on further improving the demosaicing performance of CFA 3.0. Our idea is to see if newer inpainting algorithms can help improve the overall demosaicing performance. Six conventional and deep learning based methods were compared and the FOE method yielded slight better performance than others. One key observation is that there is still room for improvement because, when we used the ground truth pan band, the overall demosaicing performance is much better than what we have right now. Hence, one future direction is to seek better inpainting methods. Another direction is to develop an end-to-end deep learning approach to demosaicing CFA 3.0.

\section{CONFLICT OF INTEREST}

The authors declare no conflict of interest.

\section{ACKNOWLEDGEMENTS}

This work was partially supported by NASA Jet Propulsion Laboratory under contract \# 80NSSC17C0035. The views, opinions and/or findings expressed are those of the author(s) and should not be interpreted as representing the official views or policies of NASA or the U.S. Government.

\section{REFERENCES}

[1] B. E. Bayer, Color imaging array. US Patent 3,971,065, July 20, 1976. 
Signal \& Image Processing: An International Journal (SIPIJ) Vol.11, No.6, December 2020

[2] J. F. Bell III, et al., "The Mars Science Laboratory Curiosity Rover Mast Camera (Mastcam) Instruments: Pre-Flight and In-Flight Calibration, Validation, and Data Archiving," AGU Journal Earth and Space Science, 2017.

[3] M. Dao, C. Kwan, B. Ayhan, and J. F. Bell, "Enhancing Mastcam Images for Mars Rover Mission," 14th International Symposium on Neural Networks, pp. 197-206, 2017.

[4] C. Kwan, B. Budavari, M. Dao, B. Ayhan, and J. F. Bell, "Pansharpening of Mastcam images," IEEE International Geoscience and Remote Sensing Symposium, pp. 5117-5120, Fort Worth, Texas, 2017.

[5] B. Ayhan, M. Dao, C. Kwan, H. Chen, J. F. Bell, and R. Kidd, "A Novel Utilization of Image Registration Techniques to Process Mastcam Images in Mars Rover with Applications to Image Fusion, Pixel Clustering, and Anomaly Detection," IEEE Journal of Selected Topics in Applied Earth Observations and Remote Sensing, 10(10), pp. 4553-4564, 2017.

[6] J. Hamilton and J. Compton, Processing color and panchromatic pixels. U.S. Patent 20070024879A1, 2007.

[7] T. Kijima, H. Nakamura, J. T. Compton, J. F.; Hamilton, and T. E. DeWeese, Image sensor with improved light sensitivity. U.S. Patent 0268 533, Nov., 2007.

[8] C. Kwan and J. Larkin, "Demosaicing of Bayer and CFA 2.0 Patterns for Low Lighting Images," Electronics, 8, 1444, 2019.

[9] C. Kwan and J. Larkin, "Comparison of Denoising Algorithms in Demosacing Low Lighting Images Using CFA 2.0,"'Signal \& Image Processing: An International Journal (SIPIJ), vol. 11, no. 5, October 29, 2020.

[10] C. Kwan and J. Larkin, "Demosaicing for Mastcam Images Using A New Color Filter Array," Signal \& Image Processing: An International Journal (SIPIJ), Vol. 11, No. 3, May 31, 2020.

[11] C. Kwan, J. Larkin, and B. Budavari, "Demosaicing of Real Low Lighting Images Using CFA 3.0," Signal \& Image Processing: An International Journal (SIPIJ), vol. 11, no. 4, August 2020.

[12] C. Kwan, J. Larkin, and B. Ayhan, "Demosaicing of CFA 3.0 with Application to Low Lighting Images," Sensors, 20(12), 3423, June 22, 2020.

[13] L. Zhang, X. Wu, A. Buades, and X. Li, "Color demosaicking by local directional interpolation and nonlocal adaptive thresholding," J. Electron. Imaging, 20, 2011.

[14] D. Doshkov, P. Ndjiki-Nya, H. Lakshman, M. Köppel, and T. Wiegand, "Towards efficient intra prediction based on image inpainting methods," 28th Picture Coding Symposium. IEEE, 2010.

[15] Inpaint_nans, https://www.mathworks.com/matlabcentral/fileexchange/4551-inpaint_nans, accessed on October 29, 2020.

[16] S. Roth and M. J. Black, "Fields of Experts," Int J Comput Vis., 82: 205, 2009.

[17] J. Yu, Z. Lin, J. Yang, X. Shen, X. Lu, and T. Huang, "Generative Image Inpainting with Contextual Attention," arXiv:1801.07892 [cs.CV]. 2018.

[18] C. Kwan, B. Chou, L. M. Kwan, and B. Budavari, "Debayering RGBW Color Filter Arrays: A Pansharpening Approach," IEEE Ubiquitous Computing, Electronics \& Mobile Communication Conference, pp. 94-100, New York City, 2017.

[19] G. Vivone, et al.,"A Critical Comparison Among Pansharpening Algorithms," IEEE Trans. Geoscience and Remote Sensing, 53(5), 2015.

[20] J. G. Liu, "Smoothing filter based intensity modulation: A spectral preserve image fusion technique for improving spatial details," Int. J. Remote Sens., 21, 18, 2000.

[21] B. Aiazzi, et al.,"MTF-tailored multiscale fusion of high-resolution MS and pan imagery," Photogramm. Eng. Remote Sens., 72(5), pp. 591-596, 2006.

[22] G. Vivone, et al., "Contrast and error-based fusion schemes for multispectral image pansharpening," IEEE Trans. Geosci. Remote Sensing Lett., 11(5), pp. 930-934, 2014.

[23] C. Laben and B. Brower, Process for enhancing the spatial resolution of multispectral imagery using pan-sharpening. U.S. Patent 6011 875, Jan. 4, 2000.

[24] B. Aiazzi, et al., "Improving component substitution pansharpening through multivariate regression of MS+pan data," IEEE Trans. Geosci. Remote Sensing, 45(10), pp. 3230-3239, 2007.

[25] W. Liao, et al., "Processing of multiresolution thermal hyperspectral and digital color data: Outcome of the 2014 IEEE GRSS data fusion contest," IEEE J. Select. Top. Appl. Earth Observ. Remote Sensing, 8, 6, 2015.

[26] J. Choi, et al., "A new adaptive component-substitution based satellite image fusion by using partial replacement," IEEE Trans. Geosci. Remote Sens., 49, 1, 2011. 
Signal \& Image Processing: An International Journal (SIPIJ) Vol.11, No.6, December 2020

[27] J. Zhou, C. Kwan, and B. Budavari, "Hyperspectral image super-resolution: A hybrid color mapping approach," Journal of Applied Remote Sensing, 10, 3, article 035024, 2016.

[28] C. Kwan, J. H. Choi, S. Chan, J. Zhou, and B. Budavai, "Resolution Enhancement for Hyperspectral Images: A Super-Resolution and Fusion Approach," IEEE International Conference on Acoustics, Speech, and Signal Processing, pp. 6180 - 6184, New Orleans, 2017.

[29] C. Kwan, B. Budavari, and G. Feng, "A Hybrid Color Mapping Approach to Fusing MODIS and Landsat Images for Forward Prediction," Remote Sensing, 10(4), 520, 2017.

[30] C. Kwan, B. Budavari, A. Bovik, and G. Marchisio, "Blind Quality Assessment of Fused WorldView-3 Images by Using the Combinations of Pansharpening and Hypersharpening Paradigms," IEEE Geoscience and Remote Sensing Letters, vol. 14, no. 10, pp. 1835-1839, Oct. 2017.

[31] C. Kwan, B. Ayhan, and B. Budavari, "Fusion of THEMIS and TES for Accurate Mars Surface Characterization," IEEE International Geoscience and Remote Sensing Symposium, pp. 3381-3384, Fort Worth, Texa, 2017.

[32] L. Zhang and $\mathrm{X}$. Wu, "Colordemosaicking via directional linear minimum mean square-error estimation," IEEE Trans. Image Processing, 14, 2167-2178, 2005.

[33] W. Lu and Y. P. Tan, "Color filter array demosaicking: New method and performance measures," IEEE Trans. on Image Processing, 12, 1194-1210, 2003.

[34] E. Dubois, "Frequency-domain methods for demosaicking of Bayer-sampled color images," IEEE Signal Proc. Letters, 12, 847-850, 2005.

[35] B. Gunturk, Y. Altunbasak, and R. M. Mersereau, "Color plane interpolation using alternating projections," IEEE Transactions on Image Processing, 11, 997-1013, 2002.

[36] X. Wu and N. Zhang, "Primary-consistent soft-decision color demosaicking for digital cameras," IEEE Trans. on Image Processing, 13, 1263-1274, 2004.

[37] C. Kwan, X. Zhu, F. Gao, B. Chou, D. Perez, J. Li, Y. Shen, and K. Koperski, "AssessmentofSpatiotemporalFusionAlgorithmsforPlanetandWorldviewImages," Sensors, 18,1051, 2018.

[38] SSIM.Availableonline:https://en.wikipedia.org/wiki/Structural_similarity.(Accessedon26April2019).

[39] K. Egiazarian, J. Astola, N. Ponomarenko, V. Lukin, F. Battisti, and M. Carli, "New full quality metrics based on HVS," Second International Workshop on Video Processing and Quality Metrics, Scottsdale, AZ, USA, 22-24 January 2006.

[40] N. Ponomarenko,F.Silvestri,K.Egiazarian,M. Carli, J. Astola, and V. Lukin, "OnbetweencoefficientcontrastmaskingofDCTbasisfunctions,"Third International Workshop on Video Processing and Quality Metrics for Consumer Electronics VPQM-07,Scottsdale,AZ,USA,25-26January2007.

[41] X. Zhang and B. A. Wandell, "A spatial extension of cielab for digital color image reproduction," SID Journal, 1997.

[42] C. Kwan, J. Larkin, B. Budavari, B. Chou, E. Shang, and T. D. Tran, "A comparison of compression codecs for maritime and sonar images in bandwidth constrained applications," Computers, 8(2), 32, April 28, 2019.

[43] C. Kwan, J. Larkin, B. Budavari, E. Shang, and T. Tran, "Perceptually Lossless Compression with Error Concealment for Periscope and Sonar Videos," Signal \& Image Processing: An International Journal (SIPIJ), vol. 10(02); pages 01-14, April 30, 2019.

\section{AUTHORS}

Chiman Kwan received his Ph.D. degree in electrical engineering from the University of Texas at Arlington in 1993. He has written one book, four book chapters, 15 patents, 70 invention disclosures, 380 technical papers in journals and conferences, and 550 technical reports. Over the past 25 years, he has been the PI/Program Manager of over 120 diverse projects with total funding exceeding 36 million dollars. He is also the founder and Chief Technology Officer of Signal Processing, Inc. and Applied Research LLC. He received numerous awards from IEEE, NASA, and some other agencies and has given several keynote speeches in several international conferences.

Jude Larkin received his B.S. in Computer Science from Franciscan University of Steubenville in 2015. $\mathrm{He}$ is a software engineer at ARLLC. He has been involved in diverse projects, including mission planning for UAVs, image fusion, image demosaicing, and remote sensing. 